\title{
Physical blood-brain barrier disruption induced by focused ultrasound does not overcome the transporter-mediated efflux of erlotinib
}

\author{
Sébastien Goutal ${ }^{\mathrm{a}, \mathrm{b}}$, Matthieu Gerstenmayer ${ }^{\mathrm{c}}$, Sylvain Auvity ${ }^{\mathrm{a}}$, Fabien Cailléa \\ Sébastien Mériaux ${ }^{\mathrm{c}}$, Irène Buvat ${ }^{\mathrm{a}}$, Benoit Larrat ${ }^{\mathrm{c}, 1}$, Nicolas Tournier ${ }^{\mathrm{a}, *, 1}$ \\ ${ }^{a}$ Imagerie Moléculaire In Vivo, IMIV, Institut des sciences du vivant Frédéric Joliot, Direction de la Recherche Fondamentale, CEA, Inserm, CNRS, Univ. Paris-Sud, \\ Université Paris Saclay, CEA-SHFJ, Orsay, France \\ ${ }^{\mathrm{b}}$ Molecular Imaging Research Center, MIRCen, Institut de Biologie François Jacob, Direction de la Recherche Fondamentale, CEA, Fontenay-Aux-Roses, France \\ ${ }^{\mathrm{c}}$ Neurospin, Institut des sciences du vivant Frédéric Joliot, Direction de la Recherche Fondamentale, CEA, Université Paris Saclay, Gif sur Yvette, France
}

\section{A R T I C L E I N F O}

\section{Keywords:}

Focused ultrasound

Tyrosine kinase inhibitors

Blood-brain barrier

Positron Emission Tomography

Brain tumor

Membrane transporter

P-glycoprotein

Breast Cancer Resistance Protein

\begin{abstract}
A B S T R A C T
Overcoming the efflux mediated by ATP-binding cassette (ABC) transporters at the blood-brain barrier (BBB) remains a challenge for the delivery of small molecule tyrosine kinase inhibitors (TKIs) such as erlotinib to the brain. Inhibition of ABCB1 and ABCG2 at the mouse BBB improved the BBB permeation of erlotinib but could not be achieved in humans. BBB disruption induced by focused ultrasound (FUS) was investigated as a strategy to overcome the efflux transport of erlotinib in vivo.

In rats, FUS combined with microbubbles allowed for a large and spatially controlled disruption of the BBB in the left hemisphere. ABCB1/ABCG2 inhibition was performed using elacridar $(10 \mathrm{mg} / \mathrm{kg}$ i.v). The brain kinetics of erlotinib was studied using ${ }^{11} \mathrm{C}$-erlotinib Positron Emission Tomography (PET) imaging in 5 groups $(n=4-5$ rats per group) including a baseline group, immediately after sonication (FUS), $48 \mathrm{~h}$ after FUS (FUS $+48 \mathrm{~h}$ ), elacridar (ELA) and their combination (FUS + ELA). BBB integrity was assessed using the Evan's Blue (EB) extravasation test. Brain exposure to ${ }^{11} \mathrm{C}$-erlotinib was measured as the area under the curve (AUC) of the brain kinetics (\% injected dose (\%ID) versus time (min)) in volumes corresponding to the disrupted (left) and the intact (right) hemispheres, respectively.

EB extravasation highlighted BBB disruption in the left hemisphere of animals of the FUS and FUS + ELA groups but not in the control and ELA groups. EB extravasation was not observed $48 \mathrm{~h}$ after FUS suggesting recovery of BBB integrity. Compared with the control group ( $\mathrm{AUC}_{\mathrm{Baseline}}=1.4 \pm 0.5 \% \mathrm{ID} \cdot \mathrm{min}$ ), physical $\mathrm{BBB}$ disruption did not impact the brain kinetics of ${ }^{11} \mathrm{C}$-erlotinib in the left hemisphere $(p>.05)$ either immediately $\left(\mathrm{AUC}_{\mathrm{FUS}}=1.2 \pm 0.1 \% \mathrm{ID} \cdot \mathrm{min}\right)$ or $48 \mathrm{~h}$ after FUS ( $\left.\mathrm{AUC}_{\mathrm{FUS}}+48 \mathrm{~h}=1.1 \pm 0.3 \% \mathrm{ID} \cdot \mathrm{min}\right)$. Elacridar similarly increased ${ }^{11} \mathrm{C}$-erlotinib brain exposure to the left hemisphere in the absence (AUC $\mathrm{ELA}=2.2 \pm 0.5 \% \mathrm{ID}$.min, $p<.001$ ) and in the presence of BBB disruption ( $\mathrm{AUC}_{\mathrm{FUS}+\mathrm{ELA}}=2.1 \pm 0.5 \%$ ID.min, $\mathrm{p}<.001$ ). AUC $_{\text {left }}$ was never significantly different from $\mathrm{AUC}_{\text {right }}(p>.05)$, in any of the tested conditions.
\end{abstract}

$\mathrm{BBB}$ integrity is not the rate limiting step for erlotinib delivery to the brain which is mainly governed by ABCmediated efflux. Efflux transport of erlotinib persisted despite BBB disruption.

\section{Introduction}

Drug delivery to the brain is often restricted by the blood-brain barrier (BBB), which makes the treatment of central nervous system (CNS) diseases extremely challenging [1]. In particular, the outcome of brain malignancies is poor as a result of restricted permeability of most chemotherapeutic agents through the BBB [2]. Entering this sanctuary site to target primary and metastatic brain tumors remains a major challenge for cancer research [3]. Improving the knowledge regarding the physiology of the BBB and how it controls brain permeation of anticancer drugs remains a critical need [4].

The BBB is created by the endothelial cells that form the walls of the brain microvessels [5]. The "physical barrier" component of the BBB results from tight junctions between adjacent endothelial cells [5]. This key feature of the BBB considerably reduces paracellular flux of solutes between the blood and the brain and forces most molecular traffic to

\footnotetext{
* Corresponding author at: CEA, DRF, JOLIOT, Service Hospitalier Frédéric Joliot, Orsay, 4, place du Général Leclerc, F-91401, France.

E-mail address: nicolas.tournier@cea.fr (N. Tournier).

${ }^{1}$ Benoit Larrat and Nicolas Tournier equally contributed to this work.
} 
take a transcellular route [6]. Passive diffusion across endothelial cell membrane is therefore assumed to be the main transport mechanism for drugs to reach the brain parenchyma [4]. The "functional barrier" component of the BBB mainly relies on the activity of membrane transporters expressed at the membranes of endothelial cells, that selectively regulate the transcellular traffic [7]. The expression and function of efflux transporters of the ATP-binding cassette (ABC) superfamily are recognized as major limitations for the brain distribution of many structurally unrelated compounds, including many anticancer agents [2]. The main $\mathrm{ABC}$-transporters at the $\mathrm{BBB}$ are the P-glycoprotein (ABCB1) and the Breast Cancer Resistance Protein (ABCG2) [8].

Despite dramatic advances in understanding the molecular basis of carcinogenesis, the development of targeted cancer therapy against CNS malignancies is hampered by the low brain permeation of most tyrosine kinase inhibitors (TKIs) [9]. Efflux transport by ABCB1 and ABCG2 was shown to be a major determinant of the brain distribution of most TKIs $[2,10]$. A wealth of preclinical research has addressed the synergistic impact of $A B C B 1$ and ABCG2 in limiting the BBB crossing of the epidermal growth factor receptor (EGFR)-targeting agent erlotinib $[11,12]$ and subsequent exposure to the brain and tumor tissue [13]. Inadequate brain exposure to erlotinib is assumed to account for the high rate of metastatic progression within the CNS during or after systemic benefit in patients who have non-small cell lung cancer with activating mutation of the EGFR gene [14]. Overexpression of EGFR is also frequently found in glioblastoma where erlotinib has been relatively ineffective [15]. Therefore, improving the delivery of small TKI molecules such as erlotinib to the tumor and brain regions surrounding the tumor is often proposed as a putative strategy to limit the progression of CNS lesions through molecularly targeted therapy [16,17].

Focused ultrasound (FUS)-based strategies have been proposed to locally and temporally enhance the delivery of various kinds of anticancer drugs into brain tissues [18]. In vitro studies have shown that FUS combined with microbubbles may improve the BBB permeation of compounds by the widening of interendothelial clefts and opening of tight-junctions, thus enabling a paracellular flux between adjacent endothelial monolayer [18-21]. Moreover, FUS may also promote the transendothelial transport: FUS-induced transcytosis and transendothelial openings have been reported, which may account for the net flux of compounds across the BBB [21,22]. Recent clinical translation has shown the feasibility of such techniques in patients with CNS malignancies [23]. So far, ABC transporter function is assumed to be a regulator of the transcellular diffusion of solutes across the "intact" BBB, thus hypothesizing that efflux transporters only work against the transcellular diffusion flux [6]. This suggests that compounds have to be constrained to cross the lipid bilayer to be effluxed by ABC-transporters. ABCB1 was consistently shown to efflux drugs directly from the membrane, rather than the aqueous phase [24]. According to this assumption, FUS-induced BBB disruption, with an opened paracellular route, may be sufficient to overwhelm and overcome the ABCB1/ ABCG2-mediated efflux of erlotinib at the BBB, thus providing an alternative strategy to locally improve erlotinib delivery to the brain [25]. Moreover, many aspects of ABCB1 structure and function were shown sensitive to the properties of the surrounding membrane [24]. It can be hypothesized that $\mathrm{ABC}$-transporter function may be modulated by BBB integrity. Recent studies have reported an impact of FUS-induced BBB disruption on the rat microvascular transcriptome [26] and a delayed decrease in ABCB1 expression in the sonicated area, 24 to $48 \mathrm{~h}$ after FUS $[27,28]$. This suggests that erlotinib, which brain distribution is mainly governed by ABC-transporter mediated efflux at the $\mathrm{BBB}$, may benefit from the immediate and/or delayed impact of FUSinduced $\mathrm{BBB}$ disruption as a strategy to locally improve its brain delivery.

Positron emission tomography (PET) imaging using radiolabeled substrates of $\mathrm{ABC}$-transporters is a powerful method to study efflux transporter function at the BBB in vivo [29]. Several ABCB1-specific probes, including ${ }^{11} \mathrm{C}$ - $\mathrm{N}$-demethyl-loperamide, have been developed to unveil and quantify the importance of this ABC-transporter in restricting the brain delivery of substrates [30]. ${ }^{11} \mathrm{C}$-erlotinib PET imaging has been used to confirm the synergistic role of ABCB1 and ABCG2 in controlling the brain kinetics of erlotinib in vivo [31], and to evaluate strategies to improve the brain exposure to this TKI in a translational research perspective $[32,33]$. Once the carrier-mediated efflux of erlotinib and ${ }^{11} \mathrm{C}-\mathrm{N}$-demethyl-loperamide is abolished, these compounds are able to cross the BBB, which contrasts with their extremely low baseline brain uptake [30,31].

This study aimed at elucidating the connection between the "physical" BBB and its "functional" efflux transport component in vivo. For the first time, we investigated FUS-induced BBB disruption as a mean to overcome efflux transporter function at the rat BBB. To that end, the neuropharmacokinetic consequences of FUS-induced BBB disruption and ABC-transporter inhibition or their combination were compared in vivo using ${ }^{11} \mathrm{C}$-erlotinib $\mathrm{PET}$ imaging. The impact of $\mathrm{BBB}$ disruption on $\mathrm{ABCB} 1$ function was specifically assessed using ${ }^{11} \mathrm{C}-\mathrm{N}$-desmethyl-loperamide PET imaging.

\section{Material and methods}

\subsection{Animals}

Adult male Rattus norvegicus Wistar rats (Janvier, France) were used for the study. Animals were housed and acclimatized for at least one week before experiment. Rats had free access to chow and water. All animal use procedures were in accordance with the recommendations of the European Community (86/809/CEE) and the French National Committees (law 87/848) for the care and use of laboratory animals. The experimental protocol was approved by a local ethics committee for animal use (APAFIS\#7466-20 $16110417049220 \mathrm{v} 2$ ).

All experiments were performed under isoflurane anesthesia. Anesthesia was induced and thereafter maintained using 3\% and $1.5-2.5 \%$ isoflurane in $\mathrm{O}_{2}$, respectively. Then, a catheter was inserted in the caudal lateral vein for the i.v administration of investigational compounds.

\subsection{Chemicals and radiochemicals}

Elacridar hydrochloride and 6-O-desmethyl erlotinib (OSI-420) were purchased from Syncom BV (The Netherlands). Elacridar for i.v injection $\left(10 \mathrm{mg} \cdot \mathrm{mL}^{-1}\right)$ was formulated using a co-solvent strategy resulting in a final tetrahydrofuran concentration of $4 \%(v / v)$ in sterile aqueous D-glucose solution at $2.5 \%(w / v)$ [34]. Tariquidar was purchased from Eras Labo (France). Tariquidar for i.v. injection (4.4 mg. $\mathrm{mL}^{-1}$ ) was prepared by dissolving tariquidar dimesylate 2.35 $\mathrm{H}_{2} \mathrm{O}(\sim 6 \mathrm{mg})$ in a $5 \%$ (w:v) glucose solution $(0.5 \mathrm{~mL})$ followed by dilution with sterile water $(0.5 \mathrm{~mL})$ [35]. Evans Blue (EB) was obtained from Sigma-Aldrich, France. Four grams of EB were dissolved in $10 \mathrm{~mL}$ sterile aqueous $\mathrm{NaCl} 0.9 \%(w / v)$. All solutions were extemporaneously prepared the day of animal experiments.

${ }^{11} \mathrm{C}$-Erlotinib was synthesized by ${ }^{11} \mathrm{C}$-methylation of OSI-420 following a previously described procedure [36]. ${ }^{11} \mathrm{C}-\mathrm{N}$-desmethyl-loperamide was synthetized as previously reported [37]. Purification of ${ }^{11} \mathrm{C}$ erlotinib and ${ }^{11} \mathrm{C}$ - $\mathrm{N}$-desmethyl-loperamide was ultimately performed using solid phase extraction with ethanol as an eluent so that radiotracer solution for intravenous (i.v.) injection into animals contained $10 \%$ ethanol $(v / v)$ in $0.9 \%$ aqueous saline. Radiotracer concentration was $\sim 50 \mathrm{MBq} / \mathrm{mL}$, radiochemical purity was $>98 \%$ and specific activity was $140-300 \mathrm{GBq} / \mu \mathrm{mol}$ and $100-200 \mathrm{GBq} / \mu \mathrm{mol}$ at the end of synthesis for ${ }^{11} \mathrm{C}$-erlotinib and ${ }^{11} \mathrm{C}-\mathrm{N}$-desmethyl-loperamide, respectively.

\subsection{Hemispheric BBB disruption using transcranial focused ultrasound}

A protocol using FUS was developed to induce a large and 
controlled 'line' shaped BBB disruption in rats in one brain hemisphere. We selected transcranial FUS conditions based on our previous work, allowing for a spatially controlled BBB disruption which lasts several hours after sonication [38-40]. The ultrasound set up was made of a single element spherically focused concave transducer (diameter of $25 \mathrm{~mm}$, focal depth $20 \mathrm{~mm}$, Imasonic, France) with a central frequency at $1.5 \mathrm{MHz}$. The transducer was calibrated in a water tank, using a 200$\mu \mathrm{m}$ calibrated hydrophone (HGL-0200, preamplifier AH-2020, Onda Corporation, USA) mounted on a micrometric 3D positioning stage. Full width half maximum (FWHM) focal spot size was measured to be $1.2 \times 1.2 \times 5.8 \mathrm{~mm}^{3}$. This transducer was fed by a programmable sinusoidal wave generator connected to a $10 \mathrm{~W}$ single channel radiofrequency linear amplifier (Image Guided Therapy, France).

Rats were installed in prone position on a dedicated bed into a stereotactic frame. The transducer holder was fixed on a rail allowing reproducible head-foot displacement over a line scanning the head of the animals in order to specifically sonicate the left hemisphere. The transducer was coupled to the shaved head of the animals with a latex balloon filled with deionized and degassed water. Acoustic gel was applied to the skin in order to ensure efficient coupling with the balloon. A $200 \mu \mathrm{L}$ i.v bolus of commercially available microbubbles (Sonovue $^{\circledast}$, Bracco, Italy) was injected. Ultrasound sonication started immediately after microbubbles injection, with continuous waves set at an estimated peak negative acoustic pressure of 0.6 MPa in situ at focus. Animal weight was accounted for in this estimation since it may impact acoustic transmission through the skull [38]. A calibration of the ultrasound beam through skulls, from rats with different body weight, was previously done in water tank. The transducer, continuously shooting the ultrasound wave, was repeatedly moved back and forth above the left hemisphere with $2 \mathrm{~s}$ repetition cycles. Under sonication, lifetime of microbubbles in the bloodstream is shorter than $5 \mathrm{~min}$ [41]. Rats were therefore sonicated $5 \mathrm{~min}$ to take maximum advantage of the presence of microbubble in the circulation. A similar protocol was already validated in rats in our previous work [39] (Fig. 1).

\subsection{Inhibition of $A B C$-transporter function at the $B B B$}

Pharmacological inhibition of ABCB1 and ABCG2 was obtained using an i.v. dose of elacridar (10 mg/kg; ELA) [31]. Elacridar was injected $2 \mathrm{~min}$ after FUS (see below) and $8 \mathrm{~min}$ before PET imaging to allow for maximal ABCB1/ABCG2 inhibition during ${ }^{11} \mathrm{C}$-erlotinib PET acquisition. ABCB1-specific inhibition was achieved using tariquidar to display the impact of decreased ABCB1 function on the brain kinetics of ${ }^{11} \mathrm{C}-\mathrm{N}$-desmethyl-loperamide [42]. Tariquidar $(8 \mathrm{mg} / \mathrm{kg})$ was administered i.v $15 \mathrm{~min}$ before PET as previously described [35].

\subsection{Checking $B B B$ integrity using $E B$ extravasation test}

All animals included in the study underwent an EB extravasation test to track any BBB disruption that may have occurred during experiments. To that end, animals were i.v. injected with $2 \mathrm{~mL} / \mathrm{kg}$ of the $4 \%$ EB solution. For animals that received FUS induced BBB disruption, EB was injected $1 \mathrm{~min}$ after ultrasound exposure. At the end of experiments, animals were decapitated and the brain was removed from the skull. BBB integrity was determined visually by three independent assayers on the intact freshly excised brain and then on $\sim 5 \mathrm{~mm}$ thick coronal gross pathology slices. Although qualitative, this method is very specific and has been widely used. Indeed, no EB extravasation is detected in the brain except where ultrasound was shot (Fig. 1).

\subsection{MRI-based evaluation of the FUS-induced BBB disruption protocol}

In preliminary experiments, the efficacy and tolerance of the FUSinduced BBB disruption protocol were validated using magnetic resonance imaging (MRI).

Two rats were injected with EB immediately after the FUS protocol and 2 other rats underwent the same EB injection after a sham ultrasound sonication. Right after the EB injection, MRI images were acquired for all 4 animals using a $7 \mathrm{~T}$ small animal MRI scanner (Bruker, Germany). $\mathrm{T}_{2}$-weighted (Rapid Acquisition with Refocused Echoes, RARE), Echo Time / Repetition Time (TE/TR) $=8.5 / 3000 \mathrm{~ms}$, RARE factor $=8$, spatial resolution $=0.125 \times 0.125 \times 1 \mathrm{~mm}^{3}, \quad$ matrix size $=256 \times 256 \times 10,4$ averages, acquisition time $=6.5 \mathrm{~min})$ and $\mathrm{T}_{2}{ }^{*}$ weighted (Multi Gradient Echo, TE/TR $=3 / 90 \mathrm{~ms}, 8$ echoes, spatial resolution $=0.125 \times 0.125 \times 1 \mathrm{~mm}^{3}$, matrix size $=256 * 140 * 12,4$ averages, acquisition time $=10 \mathrm{~min}$ ) acquisitions were performed to non-invasively ascertain the absence of both edema and hemorrhage, respectively [43].

One hour after FUS, animals were i.v. injected with $200 \mu \mathrm{L}$ of a gadolinium chelate as a contrast agent (gadoterate, Dotarem ${ }^{\circledR}$ $0,5 \mathrm{mmol} / \mathrm{mL}$, Guerbet, France). Animals were then MRI scanned again in order to i) visualize and localize the hemispheric BBB disruption, ii)

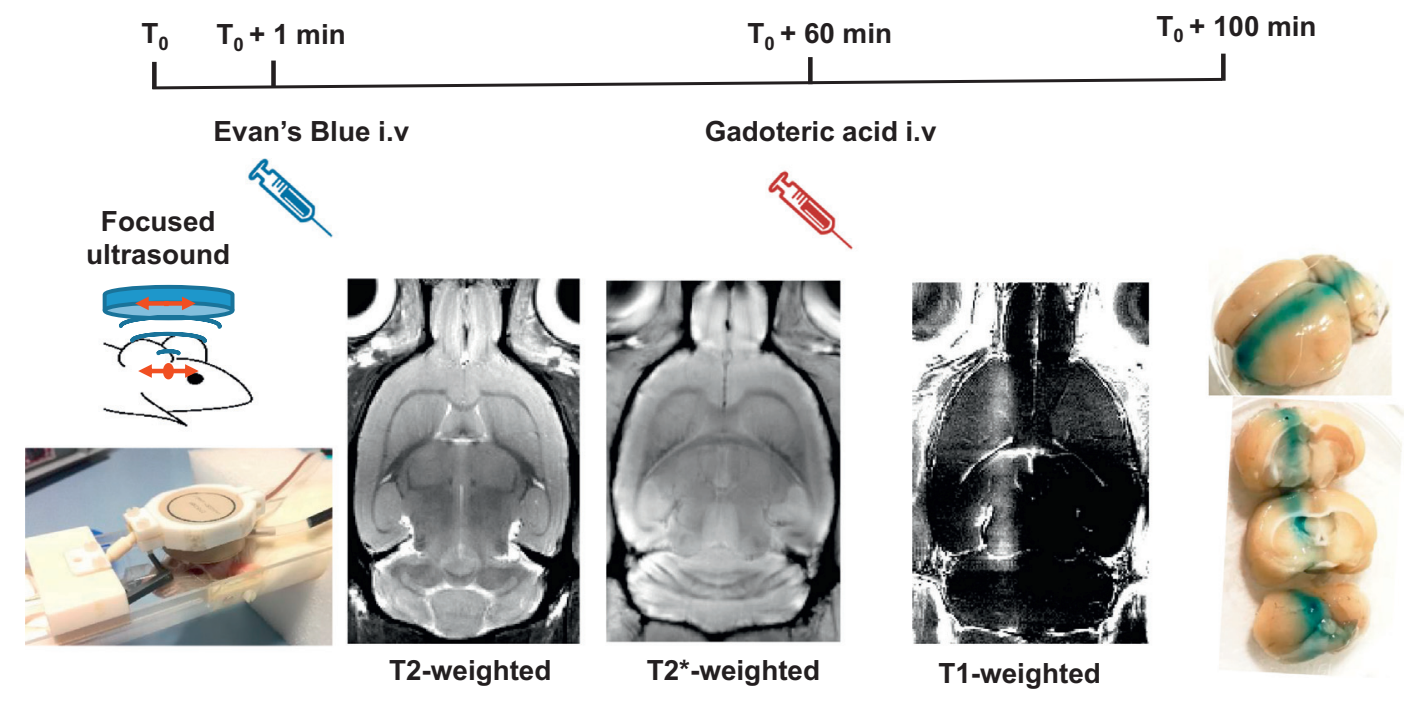

Fig. 1. MR-guided validation of the FUS-induced BBB disruption protocol.

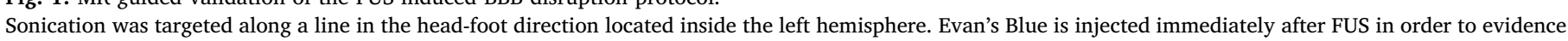

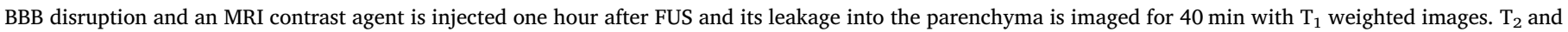
$\mathrm{T}_{2}{ }^{*}$ images do not show any tissue damage following ultrasound. 
assess whether BBB was still disrupted $60 \mathrm{~min}$ after FUS and iii) validate EB as a BBB integrity marker that could be further used in the PET study. A $\mathrm{T}_{1}$-weighted sequence (Multi Slice Multi Echo, TE/TR $=8.25$ / $300 \mathrm{~ms}$, spatial resolution $=0.125 \times 0.125 \times 1 \mathrm{~mm}^{3}$, matrix size $=256 \times 256 \times 10,10$ averages, acquisition time $=6.5 \mathrm{~min}$ ) was used to detect the signal enhancement due to gadolinium chelate delivered into brain tissues. Signal enhancement due to BBB leakage was quantified by calculating the left/right ratio of the signals in brain hemispheres.

Once the FUS protocol was validated based on MRI and EB extravasation test, we proceeded with the PET study during which no further MRI was performed except for the FUS $+48 \mathrm{~h}$ group of rats, as detailed below.

\subsection{Imaging the impact of FUS-induced BBB disruption using PET}

\subsection{1. ${ }^{11}$ C-erlotinib PET study}

Twenty rats were randomly split into four groups of five individuals. Animals of the "baseline" group were used as reference to determine the brain kinetics of erlotinib in case of intact physical and functional BBB. Animals of the "ELA" group received the ABCB1/ABCG2 inhibition protocol using i.v. elacridar. Animals of the "FUS" group underwent the FUS-induced BBB disruption in the left hemisphere. Animals of the "FUS + ELA" group had both the hemispheric FUS-induced BBB disruption and the ABCB1/ABCG2 inhibition protocol (Fig. 2).

${ }^{11} \mathrm{C}$-erlotinib PET imaging was performed after FUS, in the timewindow during which BBB disruption has been assessed $(60 \mathrm{~min}$, Fig. 1). For technical reasons and to limit radiation exposure to operators, ${ }^{11} \mathrm{C}$-erlotinib was injected as soon as possible after FUS. After FUS, all animals received EB injection as a BBB integrity marker followed by elacridar (in the ELA and FUS + ELA groups only). Animals were then transferred and installed under anesthesia from the stereotactic head-holder to the PET scanner. Due to the rapid decay of carbon11 radioactivity (half-life $=20.4 \mathrm{~min}$ ), the syringe containing ${ }^{11} \mathrm{C}$-erlotinib was extemporaneously prepared. A transmission scan was performed for attenuation correction, followed by i.v injection of ${ }^{11} \mathrm{C}$-erlotinib. The time between the end of FUS and ${ }^{11} \mathrm{C}$-erlotinib injection was standardized to $10 \mathrm{~min}$ for all groups to let enough time for operators to comfortably and reproducibly handle the procedure (Fig. 2). All groups received an equivalent dose of ${ }^{11} \mathrm{C}$-erlotinib (Table 1). PET dynamic acquisitions started immediately after ${ }^{11} \mathrm{C}$-erlotinib injection. BBB integrity was assessed at the end of the PET acquisition using the EB extravasation test (Fig.1).

\subsection{Impact of elacridar on ${ }^{11} \mathrm{C}$-erlotinib plasma kinetics}

Additional rats were used to assess the impact of elacridar on the arterial input function of ${ }^{11} \mathrm{C}$-erlotinib. Two control animals and two elacridar-treated animals were injected with ${ }^{11} \mathrm{C}$-erlotinib. Arterial plasma sampling, sample preparation and analytical methods used for the determination of parent ${ }^{11} \mathrm{C}$-erlotinib in plasma were performed as previously described [44]. Time activity curves (TACs) of unmetabolized parent ${ }^{11} \mathrm{C}$-erlotinib in plasma were expressed as the percentage of injected dose per volume (\%ID. $\mathrm{mL}^{-3}$ ) versus time. ${ }^{11} \mathrm{C}$-erlotinib plasma exposure was estimated in all tested conditions by calculating the area under the plasma TAC (AUC) from 0 to $60 \mathrm{~min}$. AUC expressed in \%ID. $\mathrm{mL}^{-3} \cdot \mathrm{min}$ is therefore inversely correlated to ${ }^{11} \mathrm{C}$-erlotinib plasma clearance $(\mathrm{Cl}=$ Dose/AUC $)$.

\subsection{Delayed impact of FUS on the brain kinetics of ${ }^{11} \mathrm{C}$-erlotinib}

Four rats have been used to investigate a putative delayed impact of FUS on BBB integrity and the brain kinetics of ${ }^{11} \mathrm{C}$-erlotinib (FUS $+48 \mathrm{~h}$ group). First, animals underwent the FUS-induced BBB disruption protocol followed by gadoterate-enhanced $\mathrm{T}_{1}$-weighted MRI (but not EB) to check BBB disruption, 60 min after FUS. Forty-eight hours after FUS, anesthesia was re-induced and animals were i.v injected by EB prior to ${ }^{11} \mathrm{C}$-erlotinib PET imaging ( $30 \mathrm{~min}$ scan). The EB extravasion test was performed at the end of PET acquisition to check BBB integrity $48 \mathrm{~h}$ after FUS.

\subsection{0. ${ }^{11} \mathrm{C}$-N-desmethyl-loperamide PET study}

The impact of $\mathrm{BBB}$ disruption on $\mathrm{ABCB} 1$ function at the $\mathrm{BBB}$ was addressed using our FUS protocol and ${ }^{11} \mathrm{C}-\mathrm{N}$-desmethyl-loperamide as an ABCB1-specific PET probe [45]. Three animals underwent FUS followed by EB injection. Ten minutes after FUS, ${ }^{11} \mathrm{C}-\mathrm{N}$-desmethyl-loperamide was i.v injected $(39.7 \pm 3.3 \mathrm{MBq})$ followed by $30 \mathrm{~min}$ PET acquisition. EB extravasion was assessed at the end of PET acquisition. Tariquidar ( $8 \mathrm{mg} / \mathrm{kg}$ i.v) was used as positive control for ABCB1 inhibition in two additional animals, as previously described [35].

\subsection{PET acquisition and data analysis}

Brain PET dynamic scans (30 or $60 \mathrm{~min}$ ) were performed under isoflurane anesthesia using an Inveon ${ }^{\circ}$ microPET system (Siemens, Germany). Images were reconstructed with the FORE + OSEM2D algorithm including normalization, attenuation, scatter and random corrections. The spatial resolution of the PET scanner is $1.5 \mathrm{~mm}$ (FWHM)

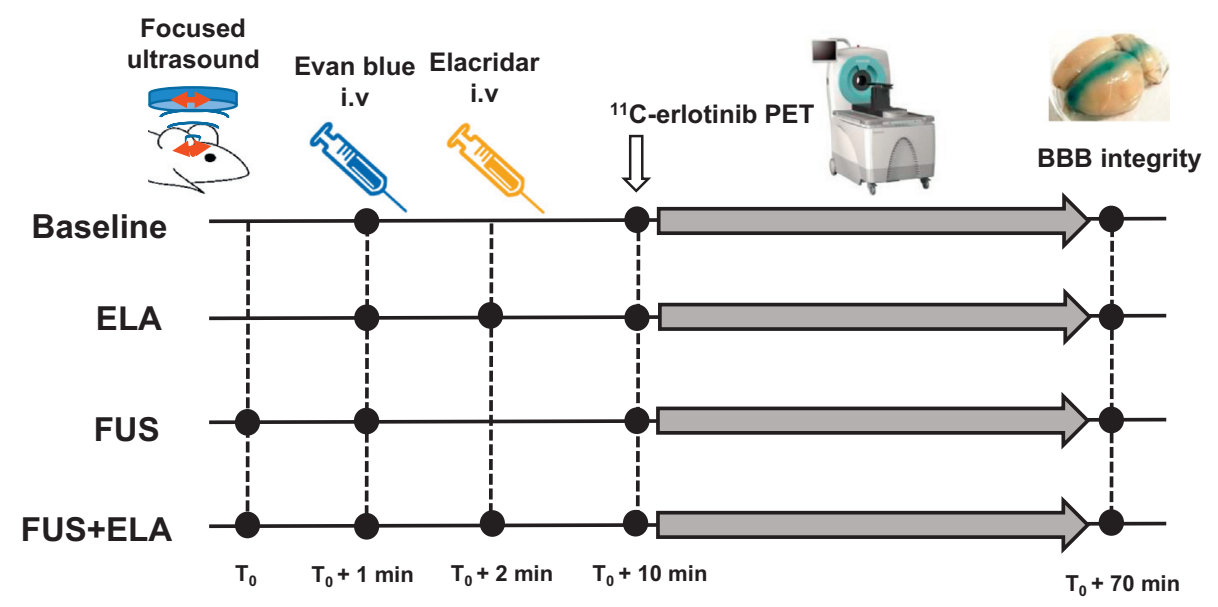

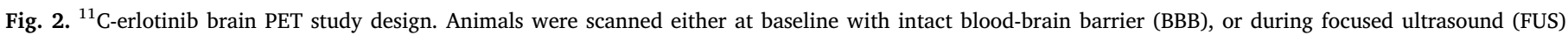
induced $\mathrm{BBB}$ disruption, $\mathrm{ABCB} 1 / \mathrm{ABCG} 2$ inhibition (ELA) or during both the FUS-induced BBB disruption and ABCB1/ABCG2 inhibition (FUS + ELA). 
Table 1

${ }^{11}$ C-erlotinib PET study data.

\begin{tabular}{|c|c|c|c|c|c|c|c|c|c|}
\hline \multirow[t]{2}{*}{ Condition } & \multirow[t]{2}{*}{$\mathrm{n}$} & \multirow{2}{*}{$\begin{array}{l}\text { Animal weight }(g \\
\text { Mean } \pm \mathrm{SD})\end{array}$} & \multirow{2}{*}{$\begin{array}{l}\text { Injected dose }{ }^{11} \mathrm{C} \text {-erlotinib (MBq; } \\
\text { Mean } \pm \mathrm{SD})\end{array}$} & \multicolumn{2}{|c|}{$\mathrm{T}_{\max }(\min ;$ Mean $\pm \mathrm{SD})$} & \multicolumn{2}{|c|}{$\mathrm{C}_{\max }\left(\% \mathrm{ID} . \mathrm{cm}^{-3} ;\right.$ Mean $\left.\pm \mathrm{SD}\right)$} & \multicolumn{2}{|c|}{ EB extravasion } \\
\hline & & & & Left & Right & Left & Right & Left & Right \\
\hline Baseline & 5 & $302 \pm 22$ & $42 \pm 9$ & $0.75 \pm 0.00$ & $0.75 \pm 0.00$ & $0.11 \pm 0.04$ & $0.10 \pm 0.03$ & 0 & 0 \\
\hline FUS & 5 & $281 \pm 14$ & $45 \pm 6$ & $0.85 \pm 0.22$ & $0.85 \pm 0.22$ & $0.11 \pm 0.01$ & $0.11 \pm 0.01$ & + & 0 \\
\hline ELA & 5 & $267 \pm 15$ & $41 \pm 5$ & $1.15 \pm 0.22$ & $1.15 \pm 0.22$ & $0.23 \pm 0.06$ & $0.25 \pm 0.07$ & 0 & 0 \\
\hline FUS + ELA & 5 & $289 \pm 18$ & $50 \pm 7$ & $1.15 \pm 0.22$ & $1.15 \pm 0.22$ & $0.19 \pm 0.03$ & $0.20 \pm 0.01$ & + & 0 \\
\hline FUS $+48 \mathrm{~h}$ & 4 & $303 \pm 8$ & $53 \pm 6$ & $0.75 \pm 0.00$ & $0.75 \pm 0.00$ & $0.13 \pm 0.05$ & $0.13 \pm 0.04$ & 0 & 0 \\
\hline
\end{tabular}

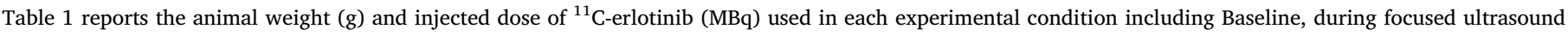

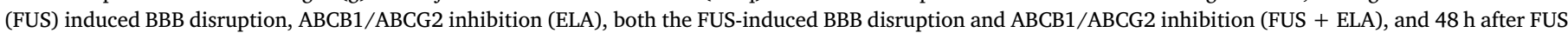

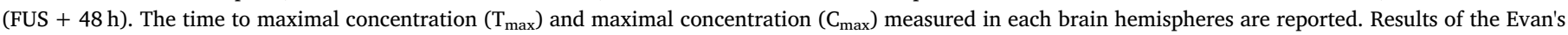
Blue (EB) extravasation test $(0=$ negative; $+=$ positive $)$ are reported for each hemisphere, in each condition.

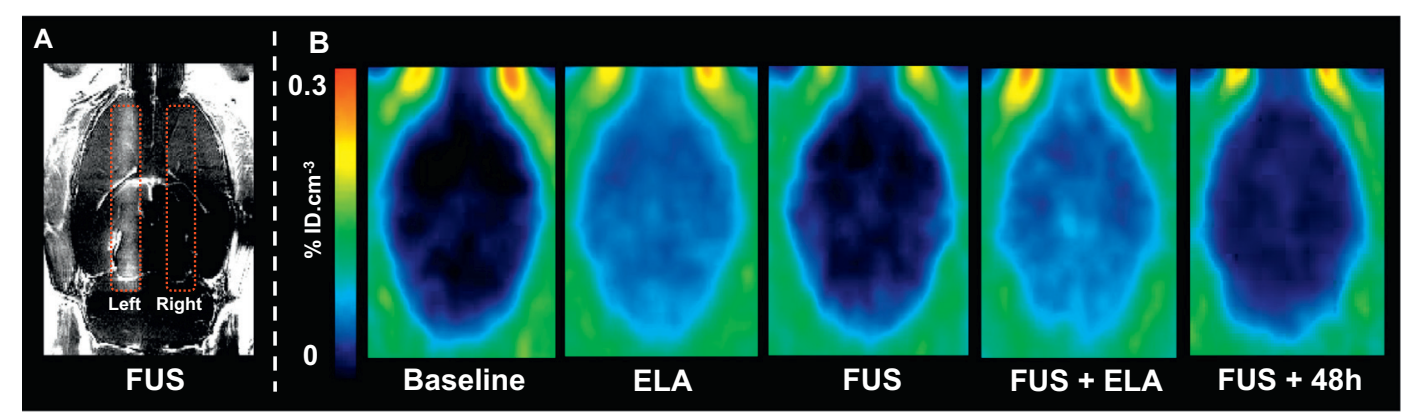

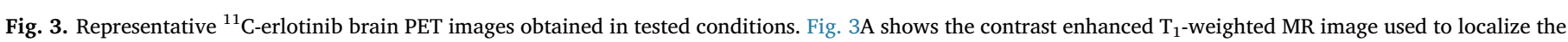

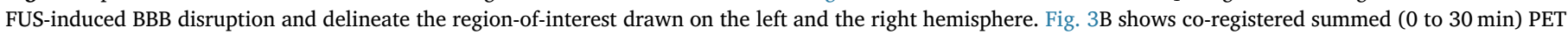

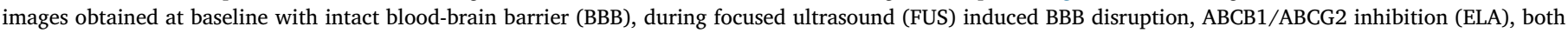

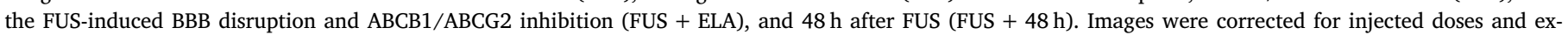
pressed as $\%$ of injected dose per volume (\%ID.cm ${ }^{-3}$ ).

\section{[46].}

Image analysis was performed using $\mathrm{PMOD}^{\circledR}$ software (version 3.8, PMOD Technologies Ltd., Switzerland). Summed PET images from 0 to 30 min were co-registered to a $\mathrm{T}_{2}$-weighted MR template built in PMOD software where a volume of interest (VOI) in the expected sonicated area of the left hemisphere has been drawn according to the MRI images obtained during preliminary experiments. The left hemisphere region was mirrored to the right hemisphere to obtain a reference VOI with intact BBB. Corresponding TACs in each VOI were generated with time frame duration of $0.25 \mathrm{~min} ; 0.5 \mathrm{~min} \times 2 ; 0.75 \mathrm{~min} ; 1 \mathrm{~min} \times 4 ; 1.5 \mathrm{~min}$; $2 \mathrm{~min} \times 4 ; 2.5 \mathrm{~min} ; 3 \mathrm{~min} \times 3 ; 3.5 \mathrm{~min} ; 4 \mathrm{~min} \times 2 ; 4.5 \mathrm{~min}$ and $5 \mathrm{~min} \times 4$. TACs were generated in these regions to describe the local kinetics of ${ }^{11} \mathrm{C}$-erlotinib, expressed as the percentage of injected dose per volume (\%ID. $\mathrm{mL}^{-3}$ ) versus time. $\mathrm{T}_{\max }$ was defined as the time at which the maximum of the TAC curve $\left(\mathrm{C}_{\max }\right)$ occurred and was used to characterize the TACs. The effect of elacridar was shown to be rapidly reversible [32]. Therefore, erlotinib exposure to each VOI was estimated as the area under the TAC (AUC) of radioactivity from 5 to $30 \mathrm{~min}$ in the region with disrupted $\left(\mathrm{AUC}_{\text {left }}\right)$ and intact $\left(\mathrm{AUC}_{\text {right }}\right) \mathrm{BBB}$.

\subsection{Statistical analysis}

All data are reported as Mean \pm 1 standard deviation (SD). PET data were compared using a two-way ANOVA with "treatment" and "hemisphere" as factors unless otherwise specified. Statistical significance was set to $p<.05$.

\section{Results}

\subsection{FUS induced a prolonged and spatially controlled BBB disruption}

$\mathrm{T}_{2}$-weighted and $\mathrm{T}_{2}{ }^{*}$-weighted images suggested the absence of FUSinduced tissue damages, such as edema or hemorrhages [43].
Subsequent $\mathrm{T}_{1}$-weighted MR images showed that the BBB was still permeable to gadoterate, at least an hour after FUS. Images highlighted a large stripe from the front of the brain to the cerebellum on a width of $\sim 2 \mathrm{~mm}$ that matches the width of the ultrasound focal spot (Fig. 1). The left-to-right hemisphere signal ratio was 2.3 and 1.7 for the two rats (Fig. 1). The volume of interest (VOI) corresponding to the disrupted BBB covered 41 to $44 \%$ of the volume of the left hemisphere. No signal enhancement could be observed in the mirrored VOI drawn in the right hemisphere, thus confirming BBB integrity. Ex vivo observation of EB extravasation performed $100 \mathrm{~min}$ after FUS was consistent in shape and volume to those observed on gadolinium enhanced MR images. No gadolinium-induced enhancement of $\mathrm{T}_{1}$-weighted $\mathrm{MR}$ images nor $\mathrm{EB}$ extravasation was observed in rats that underwent sham sonication.

\subsection{FUS-induced BBB disruption did not increase the brain exposure to ${ }^{11} \mathrm{C}$-erlotinib and ${ }^{11} \mathrm{C}$ - $\mathrm{N}$-desmethyl-loperamide}

In baseline condition, the brain distribution of ${ }^{11} \mathrm{C}$-erlotinib was low and consistent with previous ${ }^{11} \mathrm{C}$-erlotinib TACs in the brain of mice [31], nonhuman primates [32] and humans [47] (Fig. 3). ABCB1/ ABCG2 inhibition using ELA resulted in a significant $\sim 2$-fold increase in the brain maximal concentration compared with baseline in both hemispheres $(p<.05$; Fig. 4 , Table 1$)$. $\mathrm{C}_{\max }$ was achieved at $\mathrm{T}_{\max }=0.75 \pm 0.0 \mathrm{~min}$ and $1.15 \pm 0.22 \mathrm{~min}$ after the start of ${ }^{11} \mathrm{C}$-erlotinib injection in the baseline and the ELA group, respectively (Table 1). Twenty minutes post injection, the brain concentration of ${ }^{11} \mathrm{C}$-erlotinib was still significantly higher in animals who received elacridar. This difference was no longer significant after 30 min scanning, thus showing the reversibility of the ABCB1/ABCG2 inhibition effect of elacridar [32] (Fig. 4). The brain exposure to erlotinib was therefore calculated from 5 to $30 \mathrm{~min}$ and was significantly higher in the ELA group $\left(\mathrm{AUC}_{\text {left }}=2.18 \pm 0.49 \% \mathrm{ID} \cdot \mathrm{cm}^{-3} \cdot \mathrm{min}\right)$ compared with the baseline group ( $\mathrm{AUC}_{\text {left }}=1.36 \pm 0.50 \% \mathrm{ID} . \mathrm{cm}^{-3} \cdot \mathrm{min} ; p<.001$ ) 

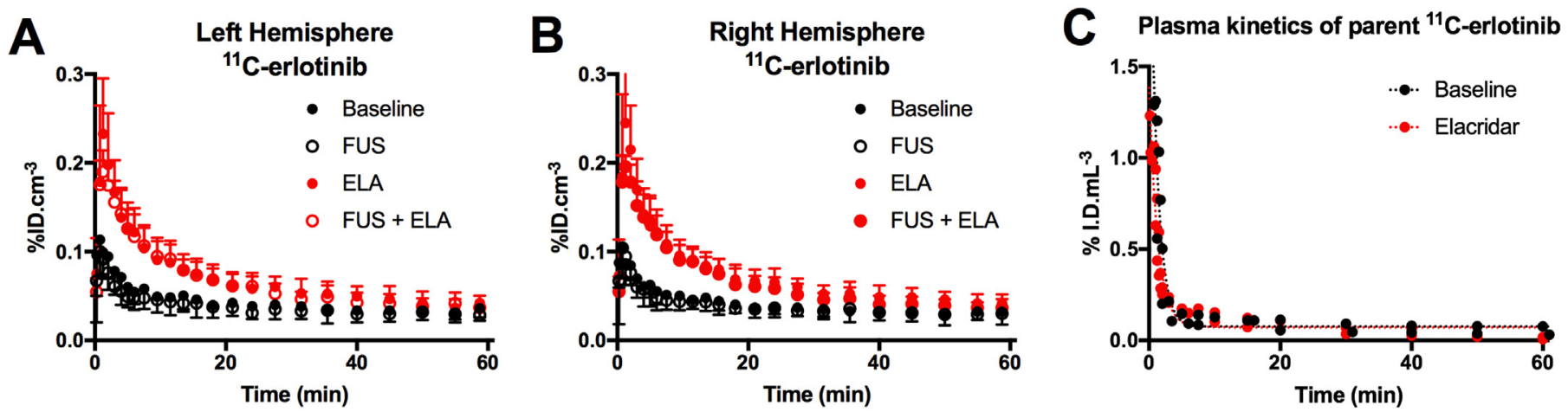

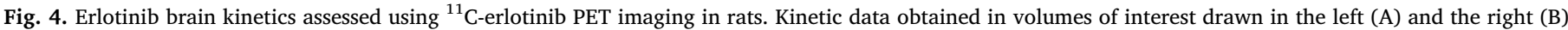

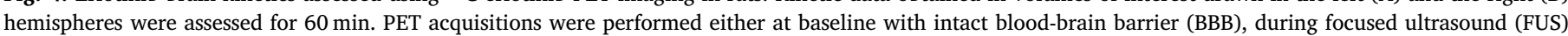

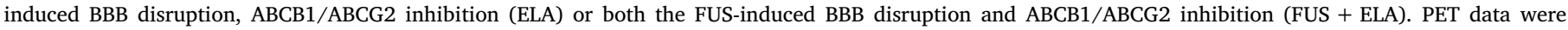

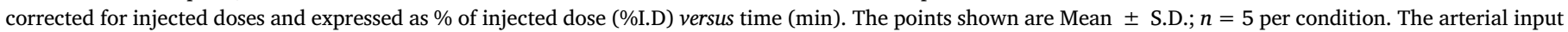
function of parent ${ }^{11} \mathrm{C}$-erlotinib in plasma in the absence and the presence of elacridar ( $n=2$ animals per condition) is shown in Fig. $4 \mathrm{C}$.

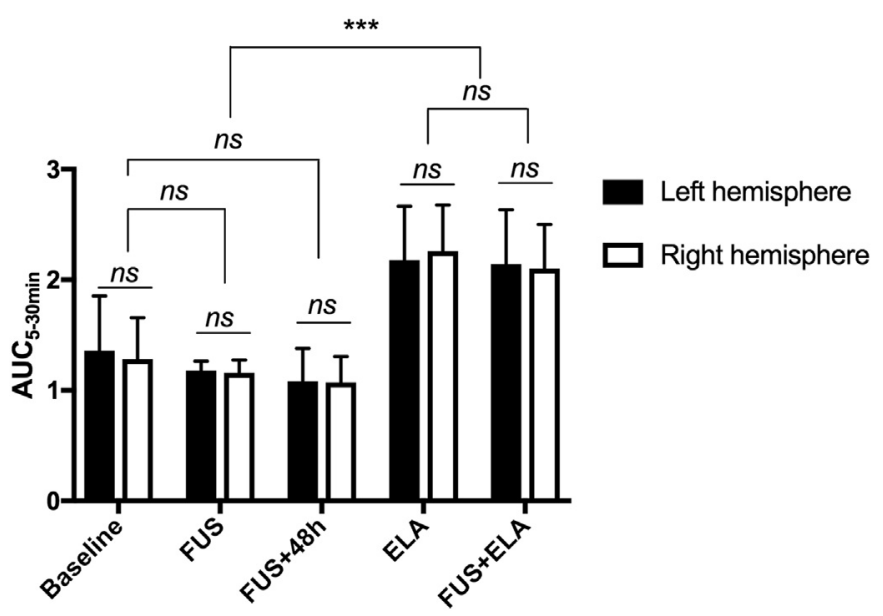

Fig. 5. ${ }^{11} \mathrm{C}$-erlotinib exposure to the brain in the presence of focused ultrasound (FUS)-induced BBB disruption and/or ABCB1/ABCG2 inhibition. PET acquisitions were performed either at baseline with intact blood-brain barrier (BBB), during focused ultrasound (FUS) induced BBB disruption, $48 \mathrm{~h}$ after FUS (FUS $+48 \mathrm{~h}$ ), after ABCB1/ABCG2 inhibition (ELA) or both the FUS-induced $\mathrm{BBB}$ disruption and $\mathrm{ABCB} 1 / \mathrm{ABCG} 2$ inhibition (FUS + ELA). Areas under the time-activity curve (AUC; \%ID.cm ${ }^{-3}$. $\mathrm{min}$ ) from 5 to $30 \mathrm{~min}$ were calculated in volumes of interest drawn in each brain hemisphere. Values are Mean \pm S.D.; $\mathrm{n}=4-5$ per condition. Statistical significance was set to $p<.05$ with $* * * p<.001: \mathrm{ns}=$ non-significant.

(Fig. 5). Neither $\mathrm{C}_{\max }$ nor $\mathrm{T}_{\max }$ were significantly different between the left and right brain hemispheres of animals of the four groups (Fig. 4, Table 1, $p>$.05).

The concentrations of ${ }^{11} \mathrm{C}$-erlotinib in plasma decreased rapidly to reach a plateau $5 \mathrm{~min}$ after injection (Fig. 4$). \mathrm{AUC}_{\text {plasma }}$ was not increased in elacridar-treated animals (6.1 and 4.5\%ID.cm $\left.{ }^{-3} . \mathrm{min}\right)$ compared with controls (4.0 and 7.7\%ID.cm ${ }^{-3} . \mathrm{min}$ ).

Hemispheric BBB disruption performed in the FUS group did not measurably impact the brain $\mathrm{C}_{\max }$ nor $\mathrm{T}_{\max }$ compared with baseline (Fig. 4, Table 1, $p>.05$ ). The brain kinetics of ${ }^{11} \mathrm{C}$-erlotinib in the FUS + ELA group were similar to those obtained in the ELA group (Fig. 4). Erlotinib exposure to the left hemisphere in the FUS + ELA group was significantly higher than baseline $(p<.001)$ and FUS $(\mathrm{p}<.001)$ but was not different from that measured in the ELA group $(\mathrm{p}>.05)$.

Animals scanned $48 \mathrm{~h}$ after FUS showed similar brain kinetics to

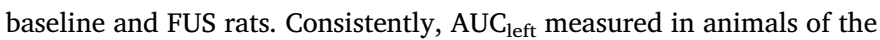
FUS $+48 \mathrm{~h}$ group was not significantly different from baseline and FUS $(p>.05)$ (Figs. 5 and 6).
The brain distribution of the ABCB1-specific PET probe ${ }^{11} \mathrm{C}-\mathrm{N}$-desmethyl-loperamide was low and consistent with previously reported PET data in rats [48]. FUS did not increase the brain exposure to the left hemisphere compared with the right hemisphere (paired $t$-test, $\mathrm{p}>$.05). ABCB1 inhibition using tariquidar substantially increased the brain uptake of ${ }^{11} \mathrm{C}-\mathrm{N}$-desmethyl-loperamide. In the right (intact) hemisphere, $\mathrm{AUC}_{\text {right }}$ was 3.2 and 3.9-fold higher in tariquidar-treated animals compared with FUS animals (Fig. 7).

In all tested conditions, $\mathrm{AUC}_{\text {left }}$ was not significantly different from $\mathrm{AUC}_{\text {right}}$, thus showing the lack of difference in the brain exposure to ${ }^{11} \mathrm{C}$-erlotinib or ${ }^{11} \mathrm{C}-\mathrm{N}$-desmethyl-loperamide between the left and the right hemisphere, regardless of the presence of FUS-induced BBB disruption or ABC-transporter inhibition (Fig. 5, p > .05).

After PET, animals were sacrificed and dissected to observe EB extravasation. EB extravasation was obvious in the left hemisphere of all animals of the FUS and FUS + ELA groups. EB extravasation highlighted a large hemispheric BBB disruption which contrasted with the absence of extravasation in the right hemisphere and in the tissue surrounding the FUS-covered volume. The mapping of EB distribution was consistent with the linear stripe shaped gadolinium contrast enhancement observed one hour after sonication in the preliminary MRI study (Fig. 1). EB extravasation was not observed in any animals of the baseline, ELA and tariquidar groups, thus confirming physical integrity of the BBB in these conditions. EB extravasation was not observed in animals of the FUS $+48 \mathrm{~h}$ group either, suggesting recovery of BBB integrity $48 \mathrm{~h}$ after FUS (Table 1 ).

\section{Discussion}

FUS-induced BBB disruption is currently developed as a muchneeded and non-invasive clinical mean for bypassing the BBB and improving drug delivery of therapeutic agents, including cytotoxic agents [1] and larger objects such as antibodies, liposomal formations or nanoparticles [18]. Compared with systemic pharmacological inhibition approaches, the FUS-induced BBB disruption allows for a brain specific and localized enhancement of drug delivery, with less peripheral toxicity $[18,49]$. It was recently shown that repeated opening of the BBB using FUS, in combination with systemic microbubble injection, is safe and well tolerated in patients [23]. We therefore investigated FUS-induced BBB disruption as a mean to improve the brain exposure to erlotinib in vivo.

To that end, we used ${ }^{11} \mathrm{C}$-erlotinib PET imaging in rats during FUSinduced $\mathrm{BBB}$ disruption and/or pharmacological inhibition of efflux transporters. FUS-induced $\mathrm{BBB}$ disruption did not improve the brain exposure to ${ }^{11} \mathrm{C}$-erlotinib while $\mathrm{ABCB} 1 / \mathrm{ABCG} 2$ inhibition did. A similar result was obtained using the ABCB1-specific PET probe ${ }^{11} \mathrm{C}-\mathrm{N}$ - 


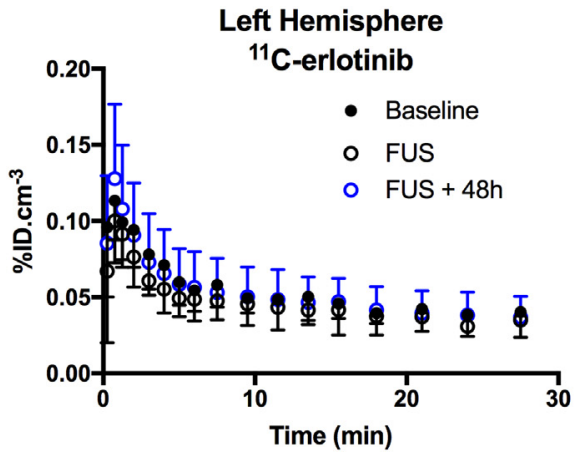

Fig. 6. Brain kinetics of ${ }^{11} \mathrm{C}$-erlotinib obtained $48 \mathrm{~h}$ after FUS-induced BBB disruption. PET data measured $48 \mathrm{~h}$ avec FUS (FUS $+48 \mathrm{~h}, \mathrm{n}=4$ ) have been acquired for $30 \mathrm{~min}$ after ${ }^{11} \mathrm{C}$-erlotinib injection. Data were corrected for injected doses and expressed as $\%$ of injected dose (\%ID.cm ${ }^{-3}$ ) versus time ( $\mathrm{min}$ ). Data can be compared with those obtained in baseline animals $(n=5)$ and during FUS $(n=5)$. The points shown are Mean \pm S.D. desmethyl-loperamide. Using this alternative PET probe, we showed that lack of effect of FUS on erlotinib brain kinetics may not restrict to dual ABCB1/ABCG2 substrates but may also concern ABCB1 substrate.

First, we used pharmacological inhibition protocols to assess the impact of $\mathrm{ABC}$-transporter function on the brain kinetics of ${ }^{11} \mathrm{C}$-erlotinib and ${ }^{11} \mathrm{C}-\mathrm{N}$-desmethyl-loperamide. Erlotinib is a substrate for both $\mathrm{ABCB} 1$ and $\mathrm{ABCG} 2$, the main efflux transporters expressed at the BBB [50]. Studies in transporter knockout mice showed that ABCB1 and ABCG2 work together in limiting brain distribution of most TKIs including erlotinib $[11,12]$. Due to the functional cooperation between $A B C B 1$ and $A B C G 2$, brain delivery of dual ABCB1/ABCG2 substrates can be significantly enhanced only when the activity of both transporters is simultaneously inhibited thus adding an extra difficulty to overcome ABC-transporter mediated efflux at the BBB [11,12].

We used a pharmacological inhibition strategy to show the impact of $\mathrm{ABCB} 1 / \mathrm{ABCG} 2$ inhibition at the $\mathrm{BBB}$ on the brain kinetics of ${ }^{11} \mathrm{C}$ - erlotinib. Elacridar is a potent ABCB1/ABCG2 inhibitor and high dose elacridar was shown to improve the brain delivery of a large number of dual ABCB1/ABCG2 substrates including erlotinib and other TKIs in mice and nonhuman primates [2,32]. Oral elacridar has been tested in humans but failed to achieve ABCB1/ABCG2 inhibition at the BBB due to insufficient plasma concentrations [51]. In our study, elacridar enhanced the brain exposure to ${ }^{11} \mathrm{C}$-erlotinib with no or a negligible impact on the plasma clearance in the limited time of PET acquisition (60 $\mathrm{min}$ ), as previously reported in other animal species and humans [31-33]. The increase in brain exposure was not therefore due to any peripheral impact of elacridar on the plasma kinetics ${ }^{11} \mathrm{C}$-erlotinib.

Previous experiments performed in mice have shown the reversibility of $A B C B 1 / A B C G 2$ inhibition using i.v bolus injected elacridar [32]. In our study, ABCB1/ABCG2 inhibition did not last $>30 \mathrm{~min}$. This suggests that repeated doses or i.v infusion of high doses of elacridar, which tolerance is not known, would be necessary to enhance
A
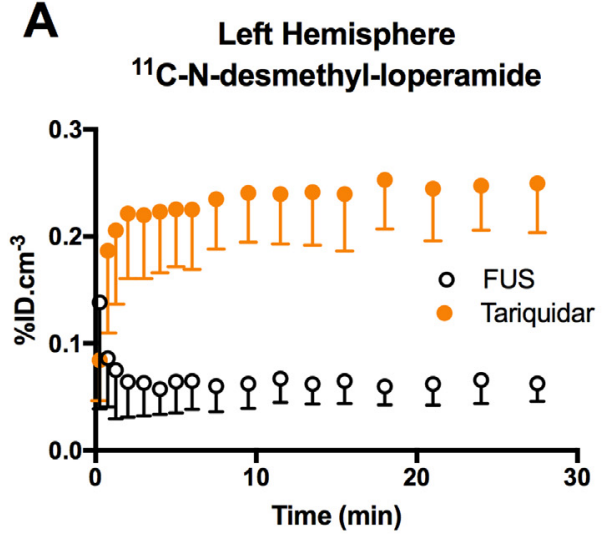

C

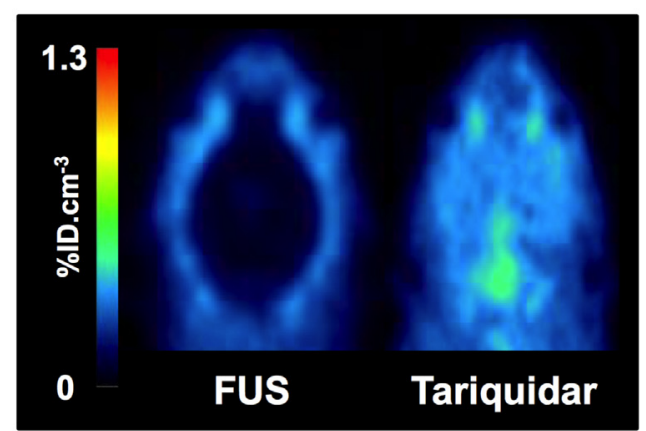

B
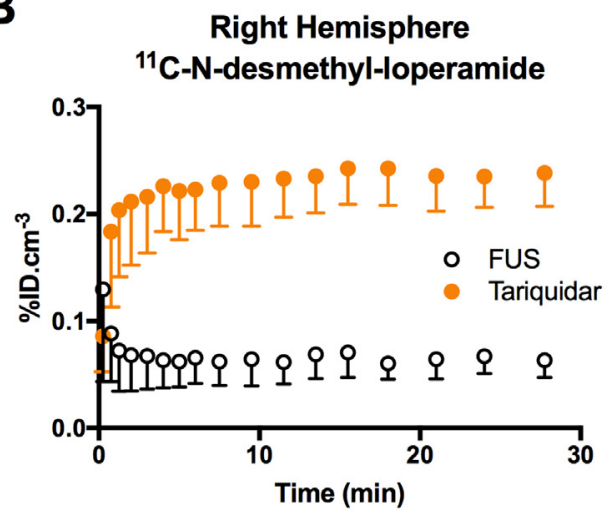

D

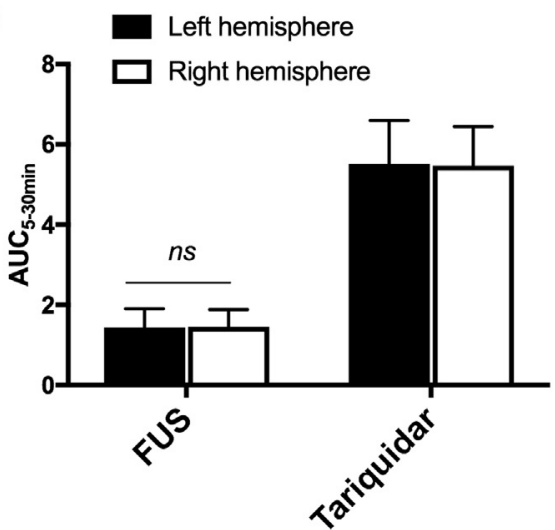

Fig. 7. Impact of FUS-induced BBB disruption and ABCB1 inhibition on the brain kinetic of ${ }^{11} \mathrm{C}-N$-desmethyl-loperamide. The brain kinetics of ${ }^{11} \mathrm{C}-\mathrm{N}$-desmethylloperamide in the left (A) and right (B) hemispheres are shown during FUS-induced BBB disruption of the left hemisphere (FUS; $n=3$ ) and after ABCB1 inhibition using tariquidar $(8 \mathrm{mg} / \mathrm{kg}$, i.v; $n=2)$ as a positive control. Corresponding summed PET images are reported in Fig. 7C. ${ }^{11} \mathrm{C}-\mathrm{N}$-desmethyl-loperamide exposure to each hemisphere $\left(\mathrm{AUC}_{5-30 \mathrm{~min}}\right.$ ) is reported in Fig. 7D. Data are Mean \pm S.D. 
the brain uptake of erlotinib and substantially engage the EGFR-kinase domain within CNS lesions in patients. In our study, a $\sim 2$-fold increase in the brain concentrations and exposure (AUC) of ${ }^{11} \mathrm{C}$-erlotinib was observed following i.v. injection of $10 \mathrm{mg} / \mathrm{kg}$ elacridar. Agarwal et al. [13] reported a 4-fold increase in the brain concentrations of erlotinib $30 \mathrm{~min}$ after jugular administration of $10 \mathrm{mg} / \mathrm{kg}$ elacridar in rats. This suggests that ${ }^{11} \mathrm{C}$-erlotinib brain uptake was not maximal in our study involving peripheral i.v. administration of a same dose.

${ }^{11} \mathrm{C}-\mathrm{N}$-desmethyl-loperamide is not transported by ABCG2 and has been developed to non-invasively and specifically study ABCB1 function at the BBB using PET [30]. ABCB1-specific inhibition at BBB has been successfully achieved using i.v tariquidar which was shown to substantially increase the brain uptake of ${ }^{11} \mathrm{C}$ - $\mathrm{N}$-desmethyl-loperamide in humans [52]. In rats, tariquidar increased the brain PET signal which confirmed the importance of ABCB1 in controlling the brain kinetics of ${ }^{11} \mathrm{C}-\mathrm{N}$-desmethyl-loperamide (Fig. 7).

Once $\mathrm{ABC}$-transporters are fully inhibited, exposure to ${ }^{11} \mathrm{C}$-erlotinib and ${ }^{11} \mathrm{C}-\mathrm{N}$-desmethyl-loperamide is similar in the brain and other tissues that are not protected by efflux transporters [32,53]. This demonstrates the ability of erlotinib and $N$-desmethyl-loperamide to freely cross the "physical" BBB, probably by passive diffusion. This property is required for PET radiotracer to yield maximal image contrast between the baseline condition and the situation where efflux transporter function is decreased, and specifically reveal the transporter-mediated efflux rather than the physical BBB [29,30,54].

We used FUS conditions with a given dose of microbubbles to induce BBB disruption for at least $1 \mathrm{~h}$ (Fig. 1). Similar microbubble dosage have been safely used in humans [55] although lower doses (4.8-8.7 mL) were sufficient for FUS-induced BBB disruption in patients [23]. Ex vivo experiments have reported that similar FUS conditions did not induce edema and hemorrhage in the sonicated brain area [56]. Much more drastic FUS conditions than ours were shown necessary to induce a mild hyperthermia in tissues [57]. In the present study, the local tolerance of our FUS protocol was assessed using $\mathrm{T}_{2}$ and $\mathrm{T}_{2}{ }^{*-}$ weighted MRI (Fig. 1). The molecular weight of gadoterate is higher than erlotinib and $N$-desmethyl-loperamide and lower than EB (MW $=960.8 \mathrm{~g} / \mathrm{mol}$ ) (Fig. 1). Gadoterate was injected $60 \mathrm{~min}$ after FUS, which confirmed that BBB was still permeable to this large BBB integrity marker, at least an hour after FUS. The first 30 min PET data were sufficient to unveil the impact of ABC-transporter function on the brain exposure to ${ }^{11} \mathrm{C}$-erlotinib and ${ }^{11} \mathrm{C}-\mathrm{N}$-desmethyl-loperamide. The FUS protocol thus offered a convenient time-window to assess the impact of BBB disruption on the brain exposure to these PET ligands that were injected only $10 \mathrm{~min}$ after FUS. Moreover, the EB extravation was assesed in each animal to check BBB integrity after PET acquisition, thus ensuring the efficacy of the FUS protocol for each experiment. The region with $\mathrm{BBB}$ disruption was larger in width, depth and length than the spatial resolution of the microPET scanner used in this study [46]. Changes in the brain kinetics of such a large region which covered $\sim 40 \%$ of the volume of the hemisphere should be detected in our PET conditions.

Fundamental physico-chemical features of compounds are related to their ability to penetrate the BBB and exhibit CNS activity. The molecular weight and lipophilicity are factors relevant to substantial BBB permeation [58] So far, criteria of selection of therapeutic compounds and objects to be delivered through FUS-induced BBB disruption are mainly based on their size and molecular weight, thus only taking their interaction with the "physical" barrier component into account $[40,59]$. Compared with gadoteric acid $(\mathrm{MW}=558.6 \mathrm{~g} / \mathrm{mol}, \log \mathrm{P}=-2.8$ [60]), erlotinib ( $\mathrm{MW}=393.4 \mathrm{~g} / \mathrm{mol}, \log \mathrm{P}=3.1$ [61]) and $N$-desmethyl-loperamide ( $\mathrm{MW}=463.0 \mathrm{~g} / \mathrm{mol}, \log \mathrm{P}=4.1$ [62]) benefit from suitable physico-chemical properties to cross biological membranes. Preclinical experiments have shown the relevance of FUS-induced BBB disruption to improve the delivery of anticancer drugs such as doxorubicine [63], methotrexate [64], cytarabine [65], carboplatine [66], temozolomide or irinotecan [67]. Interestingly, many chemotherapeutic agents are in vitro substrates of $\mathrm{ABCB} 1$ and/or ABCG2 [68]. Doxorubicin ( $M W=543 \mathrm{~g} / \mathrm{mol}, \log \mathrm{P}=1.3$ [69]) is an ABCB1 substrate with low brain uptake. FUS-induced BBB disruption was shown to enhance the brain delivery of doxorubicin (non-liposomal formulation) up 10-fold in rats [63] and 50-fold in mice [70] while Pgp-deficiency induced a minor 1.5-fold increase in its brain uptake in mice [71]. This suggests that the brain uptake of doxorubicin is predominantly controlled by the "physical" BBB rather than the "functional" BBB. FUS may thus be an efficient strategy to enhance the brain delivery of $A B C$-transporter substrates for which the rate limiting step is the "physical" BBB. Our results suggest that compounds with high passive diffusion potential, and for which $B B B$ permeation is predominantly governed by the functional efflux component of the BBB, may not benefit from FUS. In other words, we suggest that the carriermediated efflux, with respect to the passive diffusion potential, has to be considered as a new criterion for the selection of drug candidates to be delivered through FUS-aided BBB disruption.

We show that the paracellular route is not a way for erlotinib to escape from the efflux transport that persisted despite physical disruption of the $\mathrm{BBB}$. This suggests that $\mathrm{ABC}$-transporter activity at the BBB does not necessarily depend on the integrity of endothelial celllayer. The peak concentrations and the elimination kinetics of ${ }^{11} \mathrm{C}$-erlotinib from the brain were not different between the baseline and the FUS groups (Fig. 4, Table 1). This shows that ${ }^{11} \mathrm{C}$-erlotinib did not first enter the brain with the help of FUS to be then effluxed back to the plasma compartment. The lipophilic interaction of compounds with the plasma membrane was shown to be a key determinant of the carriermediated efflux of ABC-transporter substrates in cancer cells [24]. From a mechanistic point of view, erlotinib may thus show sufficient affinity for the plasma membranes of endothelial cells at the blood-brain interface to undergo active efflux, despite disrupted tight junctions between adjacent cells.

Another originality of our study was the combination of FUS with pharmacological inhibition of efflux transporters (FUS + ELA). We observed that tested elacridar dose similarly increased the brain delivery of erlotinib regardless of $\mathrm{BBB}$ integrity (ELA/ baseline $=$ ELA + FUS/FUS). This ensured that the presence of EB in the brain parenchyma following BBB disruption, did not impede ${ }^{11} \mathrm{C}$ erlotinib binding to the brain. Moreover, we showed that FUS did not further enhance the brain exposure to erlotinib in the situation of partial ABCB1/ABCG2 inhibition (FUS + ELA = ELA). The absence of additional or synergistic effect between $A B C B 1 / A B C G 2$ inhibition and $\mathrm{BBB}$ disruption confirms that $\mathrm{ABC}$-transporter function is the rate-limiting factor for erlotinib brain delivery, thus cross-validating the predominance of the "functional" component barrier rather than the "physical" component in controlling the brain penetration of this compound.

Elacridar-induced ABCB1/ABCG2 inhibition is very transient and elacridar was administered shortly before PET to get maximal effect [32,72]. It may be hypothesized that different time-points for elacridar injection, before or immediately after FUS, may enhance the ABCB1/ ABCG2 inhibitor property of this compound. Elacridar itself $(\mathrm{MW}=563.6 \mathrm{~g} / \mathrm{mol}, \log \mathrm{P}=5.6)$ [73] is a lipophilic ABCB1/ABCG2 substrate which brain permeation is mainly governed by ABCB1/ ABCG2-mediated efflux at the BBB [72]. According to our results, elacridar may therefore not benefit from FUS to reach the brain parenchyma. Moreover, high concentration of elacridar in the brain vasculature facing $\mathrm{ABC}$-transporters is expected to account for elacridar inhibition potency, rather than substantial exposure to the brain parenchyma [34].

The present study focused on the immediate consequences of a "physical" stress, allowing for a time- and space-controlled BBB disruption, on its "functional" properties, characterized by its effluxtransport ability. Interestingly, several studies suggested that FUS may not restrict to the "physical" disruption, but might also impact BBB physiology [26]. Using similar conditions to ours, Cho and colleagues 
reported a decrease in $\mathrm{ABCB} 1$ expression at the BBB of the sonicated area, $24 \mathrm{~h}$ after FUS [28]. Aryal et al., confirmed this observation and showed that the effect was maximum $48 \mathrm{~h}$ post FUS ( $\sim 50 \%$ decrease) [27]. These studies suggest a connection between the regulation of the "physical" and the "functional" components of the BBB in vivo. In our study, the brain exposure to ${ }^{11} \mathrm{C}$-erlotinib measured $48 \mathrm{~h}$ after FUS did not differ from the Baseline and the FUS group. However, it has been demonstrated that a $50 \%$ decrease in $\mathrm{ABCB} 1$ expression at the BBB may not be sufficient to enhance the brain uptake of avid ABCB1 substrates [54]. A dramatic decrease in efflux transporter expression, approaching complete depletion or inhibition may thus be necessary to enhance the brain delivery of erlotinib in vivo [33]. Moreover, ABCG2 may functionally compensate for any decline in ABCB1 as shown in ABCB1-deficient mice $[11,31]$. The putative long-term impact of FUS on the kinetics of $\mathrm{ABCB} 1$ and $\mathrm{ABCG} 2$ expression at the $\mathrm{BBB}$ remains to be assessed to conclude on the relevance of acute or repeated FUS as a strategy to enhance the brain delivery of their respective or shared substrates.

A limitation of our study is that our experiments have been performed in healthy rats and not in an animal model of brain tumor. Changes in the structure of the BBB and ABC-transporter expression in the presence of brain tumors or metastasis have been reported and are often assumed to impact the local drug disposition [74,75]. In the case of CNS brain lesions, the BBB disruption can be detected through MRI, using gadolinium as a contrast medium that does not cross the intact $\mathrm{BBB}$, as shown in the present study. Rhodamine-123 (MW $=380,8 \mathrm{~g}$ / mol, $\log P=1.0$ [76]) is a fluorescent $A B C B 1$ substrate dye. Using in situ brain perfusion, it was shown that diffusion of rhodamine-123 across the BBB was increased up to 20 -fold by ABCB1 inhibition in rats [77]. This showed the ability of Rhodamine- 123 to cross the physical $\mathrm{BBB}$ and a predominant impact of $\mathrm{ABCB} 1$ in restricting the $\mathrm{BBB}$ permeation of this compound [76]. An intact efflux function of rhodamine123 at the blood-tumor barrier was reported in a preclinical model of brain metastases despite obvious physical BBB disruption [78]. These data are consistent with our results obtained at the healthy BBB. It may therefore be hypothesized that tumor-induced BBB disruption, similar to FUS-induced BBB disruption, may have a negligible impact on the local exposure to compounds with high passive diffusion potential, whose BBB permeation is predominantly limited by ABC-mediated efflux at the BBB. This observation remains to be demonstrated in vivo for drugs such as erlotinib. ${ }^{11} \mathrm{C}$-erlotinib PET imaging may offer the opportunity to non-invasively address the impact of tumor-induced BBB disruption on the brain and tumor exposure to this compound in animal models of brain tumors and patients [29].

In our study, investigated compounds were administered as free drugs, i.e. with no carrier. FUS was shown to be a relevant strategy to selectively deliver encapsulated drugs to the brain parenchyma [79]. Moreover, nanocarrier systems have been developed to overcome the $\mathrm{ABC}$-mediated efflux at the $\mathrm{BBB}$ and improve the brain exposure to drugs [80]. This suggests that nanocarriers combined with ultrasound may be useful to bypass both the physical and the functional BBB, thus enabling the delivery of avid ABC-transporter substrates such as erlotinib to the brain, while overcoming efflux transporter-mediated multidrug resistance at the cancer-cell level [81]. However, ABC-transporter mediated efflux clearance from the brain parenchyma to the plasma compartment has been reported in different animal species $[35,82,83]$. It will be important to assess the impact of active efflux at the $\mathrm{BBB}$ and the blood-tumor barrier on tissue kinetics and therapeutic efficacy of ABC-transporter substrates delivered through the combination of ultrasound and complex drug delivery systems.

\section{Conclusion}

The brain distribution of drugs does not solely depend on the "physical" integrity of the BBB and is difficult to predict when interaction with efflux transporters of the BBB is suspected. Using PET imaging in healthy rats, we showed that FUS-induced BBB disruption did not increase the brain uptake of ${ }^{11} \mathrm{C}$-erlotinib and ${ }^{11} \mathrm{C}-\mathrm{N}$-desmethylloperamide, two compounds for which BBB permeation is restricted by ABC-mediated efflux rather than poor ability to cross the physical BBB.

A wider range of compounds with different physicochemical properties and different extent of $\mathrm{ABC}$-mediated transport could be tested to define the features of compounds for which brain distribution is enhanced by physical BBB disruption. Higher throughput in vitro models of the $\mathrm{BBB}$ and the blood-tumor barrier, with controlled membrane integrity and $\mathrm{ABC}$-transport function, may help clarify the respective role of the physical and biological mechanisms involved.

\section{Declaration of interest}

None.

\section{Grant support}

This work was performed on a platform of France Life Imaging network partly funded by grant ANR-11-INBS-0006. This work was partly funded by CEA "Technologies for Health" transverse program.

\section{Competing interests}

The authors have declared that no competing interest exists.

\section{Acknowledgements}

We thank Géraldine Pottier, Dominique Vssodovar, Maud Goislard and Martine Guillermier for their kind help on animal experiments.

\section{References}

[1] X. Dong, Current strategies for brain drug delivery, Theranostics 8 (2018) 1481-1493.

[2] S. Durmus, J.J.M.A. Hendrikx, A.H. Schinkel, Apical ABC transporters and cancer chemotherapeutic drug disposition, Adv. Cancer Res. 125 (2015) 1-41, https://doi. org/10.1016/bs.acr.2014.10.001.

[3] J.F. Deeken, W. Löscher, The blood-brain barrier and cancer: transporters, treatment, and Trojan horses, Clin. Cancer Res. 13 (2007) 1663-1674, https://doi.org/ 10.1158/1078-0432.CCR-06-2854.

[4] W.A. Banks, From blood-brain barrier to blood-brain interface: new opportunities for CNS drug delivery, Nat. Rev. Drug Discov. 15 (2016) 275-292, https://doi.org/ 10.1038/nrd.2015.21.

[5] N.J. Abbott, A.A.K. Patabendige, D.E.M. Dolman, S.R. Yusof, D.J. Begley, Structure and function of the blood-brain barrier, Neurobiol. Dis. 37 (2010) 13-25, https:// doi.org/10.1016/j.nbd.2009.07.030.

[6] M. De Bock, V. Van Haver, R.E. Vandenbroucke, E. Decrock, N. Wang, L. Leybaert, Into rather unexplored terrain-transcellular transport across the blood-brain barrier, Glia 64 (2016) 1097-1123, https://doi.org/10.1002/glia.22960.

[7] N.J. Abbott, L. Rönnbäck, E. Hansson, Astrocyte-endothelial interactions at the blood-brain barrier, Nat. Rev. Neurosci. 7 (2006) 41-53, https://doi.org/10.1038/ nrn1824.

[8] R. Shawahna, Y. Uchida, X. Declèves, S. Ohtsuki, S. Yousif, S. Dauchy, A. Jacob, F. Chassoux, C. Daumas-Duport, P.-O. Couraud, T. Terasaki, J.-M. Scherrmann, Transcriptomic and quantitative proteomic analysis of transporters and drug metabolizing enzymes in freshly isolated human brain microvessels, Mol. Pharm. 8 (2011) 1332-1341, https://doi.org/10.1021/mp200129p.

[9] P. Ballard, J.W.T. Yates, Z. Yang, D.-W. Kim, J.C.-H. Yang, M. Cantarini, K. Pickup, A. Jordan, M. Hickey, M. Grist, M. Box, P. Johnström, K. Varnäs, J. Malmquist, K.S. Thress, P.A. Jänne, D. Cross, Preclinical comparison of osimertinib with other EGFR-TKIs in EGFR-mutant NSCLC brain metastases models, and early evidence of clinical brain metastases activity, Clin. Cancer Res. 22 (2016) 5130-5140, https:// doi.org/10.1158/1078-0432.CCR-16-0399.

[10] A. Kort, S. Durmus, R.W. Sparidans, E. Wagenaar, J.H. Beijnen, A.H. Schinkel, Brain and testis accumulation of regorafenib is restricted by breast cancer resistance protein (BCRP/ABCG2) and P-glycoprotein (P-GP/ABCB1), Pharm. Res. 32 (2015) 2205-2216, https://doi.org/10.1007/s11095-014-1609-7.

[11] H. Kodaira, H. Kusuhara, J. Ushiki, E. Fuse, Y. Sugiyama, Kinetic analysis of the cooperation of P-glycoprotein (P-gp/Abcb1) and breast cancer resistance protein (Bcrp/Abcg2) in limiting the brain and testis penetration of erlotinib, flavopiridol, and mitoxantrone, J. Pharmacol. Exp. Ther. 333 (2010) 788-796, https://doi.org/ 10.1124/jpet.109.162321.

[12] N.A. de Vries, T. Buckle, J. Zhao, J.H. Beijnen, J.H.M. Schellens, O. van Tellingen, Restricted brain penetration of the tyrosine kinase inhibitor erlotinib due to the 
drug transporters P-gp and BCRP, Investig. New Drugs 30 (2012) 443-449, https:// doi.org/10.1007/s10637-010-9569-1.

[13] S. Agarwal, P. Manchanda, M.A. Vogelbaum, J.R. Ohlfest, W.F. Elmquist, Function of the blood-brain barrier and restriction of drug delivery to invasive glioma cells: findings in an orthotopic rat xenograft model of glioma, Drug Metab. Dispos. 41 (2013) 33-39, https://doi.org/10.1124/dmd.112.048322.

[14] D.R. Camidge, W. Pao, L.V. Sequist, Acquired resistance to TKIs in solid tumours: learning from lung cancer, Nat. Rev. Clin. Oncol. 11 (2014) 473-481, https://doi. org/10.1038/nrclinonc.2014.104.

[15] T. E. Taylor, F. B. Furnari, W. K. Cavenee, Targeting EGFR for treatment of glioblastoma: molecular basis to overcome resistance, Curr. Cancer Drug Targets 12 (2012) 197-209.

[16] S. Agarwal, R. Sane, R. Oberoi, J.R. Ohlfest, W.F. Elmquist, Delivery of molecularly targeted therapy to malignant glioma, a disease of the whole brain, Expert Rev. Mol. Med. 13 (2011) e17, , https://doi.org/10.1017/S1462399411001888.

[17] J. How, J. Mann, A.N. Laczniak, M.Q. Baggstrom, Pulsatile erlotinib in EGFR-positive non-small-cell lung cancer patients with leptomeningeal and brain metastases: review of the literature, Clin. Lung Cancer 18 (2017) 354-363, https://doi. org/10.1016/j.cllc.2017.01.013.

[18] O. Couture, J. Foley, N.F. Kassell, B. Larrat, J.-F. Aubry, Review of ultrasound mediated drug delivery for cancer treatment: updates from pre-clinical studies, Transl. Cancer Res. 3 (2014) 494-511, https://doi.org/10.21037/3354.

[19] H.-L. Liu, C.-H. Fan, C.-Y. Ting, C.-K. Yeh, Combining microbubbles and ultrasound for drug delivery to brain tumors: current progress and overview, Theranostics 4 (2014) 432-444, https://doi.org/10.7150/thno.8074.

[20] K. Hynynen, N. McDannold, N. Vykhodtseva, F.A. Jolesz, Noninvasive MR imagingguided focal opening of the blood-brain barrier in rabbits, Radiology 220 (2001) 640-646, https://doi.org/10.1148/radiol.2202001804.

[21] C.X. Deng, Targeted drug delivery across the blood-brain barrier using ultrasound technique, Ther. Deliv. 1 (2010) 819-848, https://doi.org/10.4155/tde.10.66.

[22] K. Hynynen, N. McDannold, H. Martin, F.A. Jolesz, N. Vykhodtseva, The threshold for brain damage in rabbits induced by bursts of ultrasound in the presence of an ultrasound contrast agent (Optison), Ultrasound Med. Biol. 29 (2003) 473-481, https://doi.org/10.1016/S0301-5629(02)00741-X.

[23] A. Carpentier, M. Canney, A. Vignot, V. Reina, K. Beccaria, C. Horodyckid, C. Karachi, D. Leclercq, C. Lafon, J.-Y. Chapelon, L. Capelle, P. Cornu, M. Sanson, K. Hoang-Xuan, J.-Y. Delattre, A. Idbaih, Clinical trial of blood-brain barrier disruption by pulsed ultrasound, Sci. Transl. Med. 8 (2016) 343re2, https://doi.org/ 10.1126/scitranslmed.aaf6086.

[24] F.J. Sharom, Complex interplay between the P-glycoprotein multidrug efflux pump and the membrane: its role in modulating protein function, Front. Oncol. 4 (2014), https://doi.org/10.3389/fonc.2014.00041.

[25] O. van Tellingen, B. Yetkin-Arik, M.C. de Gooijer, P. Wesseling, T. Wurdinger, H.E. de Vries, Overcoming the blood-brain tumor barrier for effective glioblastoma treatment, Drug Resist. Updat. 19 (2015) 1-12, https://doi.org/10.1016/j.drup. 2015.02.002.

[26] D. McMahon, R. Bendayan, K. Hynynen, Acute effects of focused ultrasound-induced increases in blood-brain barrier permeability on rat microvascular transcriptome, Sci. Rep. 7 (2017) 45657, , https://doi.org/10.1038/srep45657.

[27] M. Aryal, K. Fischer, C. Gentile, S. Gitto, Y.-Z. Zhang, N. McDannold, Effects on Pglycoprotein expression after blood-brain barrier disruption using focused ultrasound and microbubbles, PLoS One 12 (2017) e0166061, , https://doi.org/10. 1371/journal.pone.0166061.

[28] H. Cho, H.-Y. Lee, M. Han, J.-R. Choi, S. Ahn, T. Lee, Y. Chang, J. Park, Localized down-regulation of P-glycoprotein by focused ultrasound and microbubbles induced blood-brain barrier disruption in rat brain, Sci. Rep. 6 (2016) 31201, , https://doi.org/10.1038/srep31201.

[29] N. Tournier, B. Stieger, O. Langer, Imaging techniques to study drug transporter function in vivo, Pharmacol. Ther. 189 (2018) 104-122, https://doi.org/10.1016/j. pharmthera.2018.04.006.

[30] P. Kannan, C. John, S.S. Zoghbi, C. Halldin, M.M. Gottesman, R.B. Innis, M.D. Hall, Imaging the function of P-glycoprotein with radiotracers: pharmacokinetics and in vivo applications, Clin. Pharmacol. Ther. 86 (2009) 368-377, https://doi.org/10. 1038/clpt.2009.138.

[31] A. Traxl, T. Wanek, S. Mairinger, J. Stanek, T. Filip, M. Sauberer, M. Müller, C. Kuntner, O. Langer, Breast cancer resistance protein and P-glycoprotein influence in vivo disposition of 11C-erlotinib, J. Nucl. Med. 56 (2015) 1930-1936, https:// doi.org/10.2967/jnumed.115.161273.

[32] N. Tournier, S. Goutal, S. Auvity, A. Traxl, S. Mairinger, T. Wanek, O.-B. Helal, I. Buvat, M. Soussan, F. Caillé, O. Langer, Strategies to inhibit ABCB1- and ABCG2mediated efflux transport of erlotinib at the blood-brain barrier: a PET study on nonhuman primates, J. Nucl. Med. 58 (2017) 117-122, https://doi.org/10.2967/ jnumed.116.178665.

[33] R.B. Verheijen, M.M. Yaqub, E. Sawicki, O. van Tellingen, A.A. Lammertsma, B. Nuijen, J.H.M. Schellens, J.H. Beijnen, A.D.R. Huitema, N.H. Hendrikse, N. Steeghs, Molecular imaging of ABCB1/ABCG2 inhibition at the human blood brain barrier using elacridar and 11C-erlotinib PET, J. Nucl. Med. 59 (2018) 973-975, https://doi.org/10.2967/jnumed.117.195800.

[34] S. Goutal, O. Langer, S. Auvity, K. Andrieux, C. Coulon, F. Caillé, P. Gervais, S. Cisternino, X. Declèves, N. Tournier, Intravenous infusion for the controlled exposure to the dual ABCB1 and ABCG2 inhibitor elacridar in nonhuman primates, Drug Deliv. Transl. Res. 8 (2018) 536-542, https://doi.org/10.1007/s13346-0170472-6.

[35] G. Pottier, S. Marie, S. Goutal, S. Auvity, M.-A. Peyronneau, S. Stute, R. Boisgard, F. Dollé, I. Buvat, F. Caillé, N. Tournier, Imaging the impact of the P-glycoprotein (ABCB1) function on the brain kinetics of metoclopramide, J. Nucl. Med. 57 (2016)
309-314, https://doi.org/10.2967/jnumed.115.164350.

[36] I. Bahce, E.F. Smit, M. Lubberink, A.A.M. van der Veldt, M. Yaqub, A.D. Windhorst, R.C. Schuit, E. Thunnissen, D.A.M. Heideman, P.E. Postmus, A.A. Lammertsma, N.H. Hendrikse, Development of [(11)C]erlotinib positron emission tomography for in vivo evaluation of EGF receptor mutational status, Clin. Cancer Res. 19 (2013) 183-193, https://doi.org/10.1158/1078-0432.CCR-12-0289.

[37] A. Damont, S. Goutal, S. Auvity, H. Valette, B. Kuhnast, W. Saba, N. Tournier, Imaging the impact of cyclosporin A and dipyridamole on P-glycoprotein (ABCB1) function at the blood-brain barrier: a [(11)C]-N-desmethyl-loperamide PET study in nonhuman primates, Eur. J. Pharm. Sci. 91 (2016) 98-104, https://doi.org/10. 1016/j.ejps.2016.06.005.

[38] M. Gerstenmayer, B. Fellah, R. Magnin, E. Selingue, B. Larrat, Acoustic transmission factor through the rat skull as a function of body mass, frequency and position, Ultrasound Med. Biol. (2018), https://doi.org/10.1016/j.ultrasmedbio.2018.06. 005.

[39] R. Magnin, F. Rabusseau, F. Salabartan, S. Mériaux, J.-F. Aubry, D. Le Bihan, E. Dumont, B. Larrat, Magnetic resonance-guided motorized transcranial ultrasound system for blood-brain barrier permeabilization along arbitrary trajectories in rodents, J. Ther. Ultrasound. 3 (2015) 22, , https://doi.org/10.1186/s40349-0150044-5.

[40] B. Marty, B. Larrat, M. Van Landeghem, C. Robic, P. Robert, M. Port, D. Le Bihan, M. Pernot, M. Tanter, F. Lethimonnier, S. Mériaux, Dynamic study of blood-brain barrier closure after its disruption using ultrasound: a quantitative analysis, J. Cereb. Blood Flow Metab. 32 (2012) 1948-1958, https://doi.org/10.1038/jcbfm. 2012.100 .

[41] S.-H. Hung, C.-K. Yeh, T.-H. Tsai, T. Chen, R.-C. Chen, A simple method for quantifying ultrasound-triggered microbubble destruction, Ultrasound Med. Biol. 37 (2011) 949-957, https://doi.org/10.1016/j.ultrasmedbio.2011.03.005.

[42] P. Kannan, S. Telu, S. Shukla, S.V. Ambudkar, V.W. Pike, C. Halldin, M.M. Gottesman, R.B. Innis, M.D. Hall, The "specific" P-glycoprotein inhibitor Tariquidar is also a substrate and an inhibitor for breast cancer resistance protein (BCRP/ABCG2), ACS Chem. Neurosci. 2 (2011) 82-89, https://doi.org/10.1021/ cn100078a.

[43] T. Kobus, N. Vykhodtseva, M. Pilatou, Y. Zhang, N. McDannold, Safety validation of repeated blood-brain barrier disruption using focused ultrasound, Ultrasound Med. Biol. 42 (2016) 481-492, https://doi.org/10.1016/j.ultrasmedbio.2015.10.009.

[44] D. Amor, S. Goutal, S. Marie, F. Caillé, M. Bauer, O. Langer, S. Auvity, N. Tournier, Impact of rifampicin-inhibitable transport on the liver distribution and tissue kinetics of erlotinib assessed with PET imaging in rats, EJNMMI Res. 8 (2018) 81, , https://doi.org/10.1186/s13550-018-0434-0.

[45] P. Kannan, K.R. Brimacombe, S.S. Zoghbi, J.-S. Liow, C. Morse, A.K. Taku, V.W. Pike, C. Halldin, R.B. Innis, M.M. Gottesman, M.D. Hall, N-desmethyl-loperamide is selective for P-glycoprotein among three ATP-binding cassette transporters at the blood-brain barrier, Drug Metab. Dispos. 38 (2010) 917-922, https://doi. org/10.1124/dmd.109.031161.

[46] E.P. Visser, J.A. Disselhorst, M. Brom, P. Laverman, M. Gotthardt, W.J.G. Oyen, O.C. Boerman, Spatial resolution and sensitivity of the Inveon small-animal PET scanner, J. Nucl. Med. 50 (2009) 139-147, https://doi.org/10.2967/jnumed.108. 055152.

[47] B. Weber, M. Winterdahl, A. Memon, B.S. Sorensen, S. Keiding, L. Sorensen, E. Nexo, P. Meldgaard, Erlotinib accumulation in brain metastases from non-small cell lung cancer: visualization by positron emission tomography in a patient harboring a mutation in the epidermal growth factor receptor, J. Thorac. Oncol. 6 (2011) 1287-1289, https://doi.org/10.1097/JTO.0b013e318219ab87.

[48] M.D. Farwell, D.J. Chong, Y. Iida, S.A. Bae, B. Easwaramoorthy, M. Ichise, Imaging P-glycoprotein function in rats using [(11)C]-N-desmethyl-loperamide, Ann. Nucl. Med. 27 (2013) 618-624, https://doi.org/10.1007/s12149-013-0725-5.

[49] A. Dréan, L. Goldwirt, M. Verreault, M. Canney, C. Schmitt, J. Guehennec, J.Y. Delattre, A. Carpentier, A. Idbaih, Blood-brain barrier, cytotoxic chemotherapies and glioblastoma, Expert. Rev. Neurother. 16 (2016) 1285-1300, https://doi.org/ 10.1080/14737175.2016.1202761.

[50] S. Marchetti, N.A. de Vries, T. Buckle, M.J. Bolijn, M.A.J. van Eijndhoven, J.H. Beijnen, R. Mazzanti, O. van Tellingen, J.H.M. Schellens, Effect of the ATPbinding cassette drug transporters ABCB1, ABCG2, and ABCC2 on erlotinib hydrochloride (Tarceva) disposition in in vitro and in vivo pharmacokinetic studies employing Bcrp1 - / - Mdr1a/1b - / - (triple-knockout) and wild-type mice, Mol. Cancer Ther. 7 (2008) 2280-2287, https://doi.org/10.1158/1535-7163.MCT-072250.

[51] E. Sawicki, R.B. Verheijen, A.D.R. Huitema, O. van Tellingen, J.H.M. Schellens, B. Nuijen, J.H. Beijnen, N. Steeghs, Clinical pharmacokinetics of an amorphous solid dispersion tablet of elacridar, Drug Deliv. Transl. Res. 7 (2017) 125-131, https://doi.org/10.1007/s13346-016-0346-3.

[52] W.C. Kreisl, R. Bhatia, C.L. Morse, A.E. Woock, S.S. Zoghbi, H.U. Shetty, V.W. Pike, R.B. Innis, Increased permeability-glycoprotein inhibition at the human blood-brain barrier can be safely achieved by performing PET during peak plasma concentrations of tariquidar, J. Nucl. Med. 56 (2015) 82-87, https://doi.org/10.2967/ jnumed.114.146894.

[53] J.-S. Liow, W. Kreisl, S.S. Zoghbi, N. Lazarova, N. Seneca, R.L. Gladding, A. Taku, P. Herscovitch, V.W. Pike, R.B. Innis, P-glycoprotein function at the blood-brain barrier imaged using 11C-N-desmethyl-loperamide in monkeys, J. Nucl. Med. 50 (2009) 108-115, https://doi.org/10.2967/jnumed.108.056226.

[54] T. Wanek, K. Römermann, S. Mairinger, J. Stanek, M. Sauberer, T. Filip, A. Traxl, C. Kuntner, J. Pahnke, F. Bauer, T. Erker, W. Löscher, M. Müller, O. Langer, Factors governing P-glycoprotein-mediated drug-drug interactions at the blood-brain barrier measured with positron emission tomography, Mol. Pharm. 12 (2015) 3214-3225, https://doi.org/10.1021/acs.molpharmaceut.5b00168. 
[55] D. Bokor, J.B. Chambers, P.J. Rees, T.G. Mant, F. Luzzani, A. Spinazzi, Clinical safety of SonoVue, a new contrast agent for ultrasound imaging, in healthy volunteers and in patients with chronic obstructive pulmonary disease, Investig. Radiol. 36 (2001) 104-109.

[56] H.-L. Liu, Y.-Y. Wai, W.-S. Chen, J.-C. Chen, P.-H. Hsu, X.-Y. Wu, W.-C. Huang, T.C. Yen, J.-J. Wang, Hemorrhage detection during focused-ultrasound induced blood-brain-barrier opening by using susceptibility-weighted magnetic resonance imaging, Ultrasound Med. Biol. 34 (2008) 598-606, https://doi.org/10.1016/j. ultrasmedbio.2008.01.011.

[57] T. Boissenot, A. Bordat, B. Larrat, M. Varna, H. Chacun, A. Paci, V. Poinsignon, E. Fattal, N. Tsapis, Ultrasound-induced mild hyperthermia improves the anticancer efficacy of both Taxol $^{\circledR}$ and paclitaxel-loaded nanocapsules, J. Control. Release 264 (2017) 219-227, https://doi.org/10.1016/j.jconrel.2017.08.041.

[58] H. Pajouhesh, G.R. Lenz, Medicinal chemical properties of successful central nervous system drugs, NeuroRx 2 (2005) 541-553.

[59] X. Wang, Y. Jia, P. Wang, Q. Liu, H. Zheng, Current status and future perspectives of sonodynamic therapy in glioma treatment, Ultrason. Sonochem. 37 (2017) 592-599, https://doi.org/10.1016/j.ultsonch.2017.02.020.

[60] S. Bussi, A. Coppo, C. Botteron, V. Fraimbault, A. Fanizzi, E. De Laurentiis, S. Colombo Serra, M.A. Kirchin, F. Tedoldi, F. Maisano, Differences in gadolinium retention after repeated injections of macrocyclic MR contrast agents to rats, J. Magn. Reson. Imaging 47 (2018) 746-752, https://doi.org/10.1002/jmri.25822.

[61] T. Minematsu, K.M. Giacomini, Interactions of tyrosine kinase inhibitors with organic cation transporters and multidrug and toxic compound extrusion proteins, Mol. Cancer Ther. 10 (2011) 531-539, https://doi.org/10.1158/1535-7163.MCT10-0731.

[62] N. Lazarova, S.S. Zoghbi, J. Hong, N. Seneca, E. Tuan, R.L. Gladding, J.-S. Liow, A. Taku, R.B. Innis, V.W. Pike, Synthesis and evaluation of [N-methyl-11C]N-desmethyl-loperamide as a new and improved PET radiotracer for imaging P-gp function, J. Med. Chem. 51 (2008) 6034-6043, https://doi.org/10.1021/ jm800510m.

[63] J. Park, M. Aryal, N. Vykhodtseva, Y.-Z. Zhang, N. McDannold, Evaluation of permeability, doxorubicin delivery, and drug retention in a rat brain tumor model after ultrasound-induced blood-tumor barrier disruption, J. Control. Release 250 (2017) 77-85, https://doi.org/10.1016/j.jconrel.2016.10.011.

[64] J. Mei, Y. Cheng, Y. Song, Y. Yang, F. Wang, Y. Liu, Z. Wang, Experimental study on targeted methotrexate delivery to the rabbit brain via magnetic resonance imagingguided focused ultrasound, J. Ultrasound Med. 28 (2009) 871-880.

[65] H.-Q. Zeng, L. Lü, F. Wang, Y. Luo, S.-F. Lou, Focused ultrasound-induced bloodbrain barrier disruption enhances the delivery of cytarabine to the rat brain, $\mathrm{J}$. Chemother. 24 (2012) 358-363, https://doi.org/10.1179/1973947812Y. 0000000043.

[66] L. Goldwirt, M. Canney, C. Horodyckid, J. Poupon, S. Mourah, A. Vignot, J.Y. Chapelon, A. Carpentier, Enhanced brain distribution of carboplatin in a primate model after blood-brain barrier disruption using an implantable ultrasound device, Cancer Chemother. Pharmacol. 77 (2016) 211-216, https://doi.org/10.1007/ s00280-015-2930-5.

[67] K. Beccaria, M. Canney, L. Goldwirt, C. Fernandez, J. Piquet, M.-C. Perier, C. Lafon, J.-Y. Chapelon, A. Carpentier, Ultrasound-induced opening of the blood-brain barrier to enhance temozolomide and irinotecan delivery: an experimental study in rabbits, J. Neurosurg. 124 (2016) 1602-1610, https://doi.org/10.3171/2015.4 JNS142893.

[68] R.W. Robey, K.M. Pluchino, M.D. Hall, A.T. Fojo, S.E. Bates, M.M. Gottesman, Revisiting the role of ABC transporters in multidrug-resistant cancer, Nat. Rev. Cancer (2018) 1, https://doi.org/10.1038/s41568-018-0005-8.

[69] S. Cai, S. Thati, T.R. Bagby, H.-M. Diab, N.M. Davies, M.S. Cohen, M.L. Forrest, Localized doxorubicin chemotherapy with a biopolymeric nanocarrier improves survival and reduces toxicity in xenografts of human breast cancer, J. Control.
Release 146 (2010) 212-218, https://doi.org/10.1016/j.jconrel.2010.04.006.

[70] S. Alli, C.A. Figueiredo, B. Golbourn, N. Sabha, M.Y. Wu, A. Bondoc, A. Luck, D Coluccia, C. Maslink, C. Smith, H. Wurdak, K. Hynynen, M. O'Reilly, J.T. Rutka, Brainstem blood brain barrier disruption using focused ultrasound: a demonstration of feasibility and enhanced doxorubicin delivery, J. Control. Release 281 (2018) 29-41. doi:https://doi.org/10.1016/j.jconrel.2018.05.005.

[71] S. Cisternino, C. Rousselle, C. Dagenais, J.M. Scherrmann, Screening of multidrugresistance sensitive drugs by in situ brain perfusion in P-glycoprotein-deficient mice, Pharm. Res. 18 (2001) 183-190.

[72] J.P. Bankstahl, M. Bankstahl, K. Römermann, T. Wanek, J. Stanek, A.D. Windhorst, M. Fedrowitz, T. Erker, M. Müller, W. Löscher, O. Langer, C. Kuntner, Tariquidar and elacridar are dose-dependently transported by P-glycoprotein and Bcrp at the blood-brain barrier: a small-animal positron emission tomography and in vitro study, Drug Metab. 41 (2013) 754-762, https://doi.org/10.1124/dmd.112.049148.

[73] R. Sane, S. Agarwal, W.F. Elmquist, Brain distribution and bioavailability of elacridar after different routes of administration in the mouse, Drug Metab. Dispos. 40 (2012) 1612-1619, https://doi.org/10.1124/dmd.112.045930.

[74] D.S. Miller, Regulation of ABC transporters at the blood-brain barrier, Clin. Pharmacol. Ther. 97 (2015) 395-403, https://doi.org/10.1002/cpt.64.

[75] L.T. Lyle, P.R. Lockman, C.E. Adkins, A.S. Mohammad, E. Sechrest, E. Hua, D. Palmieri, D.J. Liewehr, S.M. Steinberg, W. Kloc, E. Izycka-Swieszewska, R. Duchnowska, N. Nayyar, P.K. Brastianos, P.S. Steeg, B. Gril, Alterations in pericyte subpopulations are associated with elevated blood-tumor barrier permeability in experimental brain metastasis of breast cancer, Clin. Cancer Res. 22 (2016) 5287-5299, https://doi.org/10.1158/1078-0432.CCR-15-1836.

[76] M. Duvvuri, Y. Gong, D. Chatterji, J.P. Krise, Weak base permeability characteristics influence the intracellular sequestration site in the multidrug-resistant human leukemic cell line HL-60, J. Biol. Chem. 279 (2004) 32367-32372, https://doi.org/ 10.1074/jbc.M400735200.

[77] R.K. Mittapalli, V.K. Manda, K.A. Bohn, C.E. Adkins, P.R. Lockman, Quantitative fluorescence microscopy provides high resolution imaging of passive diffusion and P-gp mediated efflux at the in vivo blood-brain barrier, J. Neurosci. Methods 219 (2013) 188-195, https://doi.org/10.1016/j.jneumeth.2013.07.001.

[78] C.E. Adkins, R.K. Mittapalli, V.K. Manda, M.I. Nounou, A.S. Mohammad, T.B. Terrell, K.A. Bohn, C. Yasemin, T.R. Grothe, J.A. Lockman, P.R. Lockman, Pglycoprotein mediated efflux limits substrate and drug uptake in a preclinical brain metastases of breast cancer model, Front. Pharmacol. 4 (2013) 136, , https://doi. org/10.3389/fphar.2013.00136.

[79] T. Boissenot, A. Bordat, E. Fattal, N. Tsapis, Ultrasound-triggered drug delivery for cancer treatment using drug delivery systems: from theoretical considerations to practical applications, J. Control. Release 241 (2016) 144-163, https://doi.org/10 1016/j.jconrel.2016.09.026.

[80] A.R. Khan, X. Yang, M. Fu, G. Zhai, Recent progress of drug nanoformulations targeting to brain, J. Control. Release (2018), https://doi.org/10.1016/j.jconrel. 2018.10.004.

[81] R. Li, Y. Xie, Nanodrug delivery systems for targeting the endogenous tumor microenvironment and simultaneously overcoming multidrug resistance properties, $\mathrm{J}$. Control. Release 251 (2017) 49-67, https://doi.org/10.1016/j.jconrel.2017.02. 020 .

[82] S. Auvity, F. Caillé, S. Marie, C. Wimberley, M. Bauer, O. Langer, I. Buvat, S. Goutal, N. Tournier, P-glycoprotein (ABCB1) inhibits the influx and increases the efflux of 11C-metoclopramide across the blood-brain barrier: a PET study on non-human primates, J. Nucl. Med. 59 (2018) 1609-1615, https://doi.org/10.2967/jnumed. 118.210104.

[83] S. Agarwal, W.F. Elmquist, Insight into the cooperation of P-glycoprotein (ABCB1) and breast cancer resistance protein (ABCG2) at the blood-brain barrier: a case study examining sorafenib efflux clearance, Mol. Pharm. 9 (2012) 678-684, https://doi.org/10.1021/mp200465c. 